Original Research Paper

\title{
Responses of Rice to Green Manure and Nitrogen Fertilizer Application
}

\author{
${ }^{1}$ Md Safiqul Islam, ${ }^{2}$ Nitish Kumar Paul, ${ }^{1}$ Md Robiul Alam, \\ ${ }^{3}$ Md Romij Uddin, ${ }^{3}$ Uttam Kumer Sarker, ${ }^{4}$ Md Ariful Islam and ${ }^{5}$ Sang Un Park \\ ${ }^{1}$ On-Farm Research Division, Region 1, Bangladesh Agricultural Research Institute, Pabna, Bangladesh \\ ${ }^{2}$ Department of Botany, University of Rajshahi, Rajshahi, Bangladesh \\ ${ }^{3}$ Department of Agronomy, Bangladesh Agricultural University, Mymensingh, Bangladesh \\ ${ }^{4}$ Pulse Research Centre, Bangladesh Agricultural Research Institute, Ishurdi, Pabna, Bangladesh \\ ${ }^{5}$ Department of Crop Science, Chungnam National University, Korea
}

Article history

Received: 01-05-2015

Revised: $15-06-2015$

Accepted: 07-07-2015

Corresponding Author:

Sang Un Park

Department of Crop Science,

Chungnam National

University, Korea

E-mail: supark@cnu.ac.kr

\begin{abstract}
The effect of green manure and nitrogen fertilizer applications on rice performance and post-harvest nutrient status of the soil was studied at the Regional Wheat Research Station of Bangladesh Agricultural Research Institute (BARI), Shyampur, Rajshahi, Bangladesh. The experiment comprised two sets of treatments: (i) green manure application (Crotalaria jusncea, Sesbania aculeata, Sesbania rostrata,Vigna radiata, and Phaseolus mungo) and (ii) application of different nitrogen levels $(0,40,80$, and $120 \mathrm{~kg}$ $\left.\mathrm{ha}^{-1}\right)$. Both treatments had a significant effect on the growth and yield of transplanted aman (T. aman) rice. We show that soil fertility can be maintained and rice yield can be improved through incorporation of green manuring and nitrogen fertilizer management. Green manure and nitrogen management generated positive responses in important morpho-physiological traits such as leaf area index (LAI), specific leaf area (SLA), crop growth rate (CGR), leaf relative growth rate (LRGR), leaf weight ratio (LWR), and net assimilation rate (NAR), which may result in a greater contribution of yielddetermining traits and eventually higher grain yield. The results indicate that all green manures in combination with higher $\mathrm{N}$ levels accelerate SLA, LWR, LAI and CGR. Better performance of potential yield-contributing characters and relatively higher nitrogen uptake and higher $\mathrm{N}$ and protein content in grain and straw were found in combination with all green manure and higher $\mathrm{N}$ level treatments. An increasing level of post-harvest soil nitrogen content was observed with the application of higher levels of nitrogen in combination with green manures. Therefore, green manuring in combination with judicial nitrogen fertilizer management is deemed necessary for sustainable $T$. aman rice cultivation.
\end{abstract}

Keywords: Green Manure, Nitrogen, Growth, Yield, T. Aman Rice

\section{Introduction}

The inclusion of summer mungbean, with or without its residue, in a rice-wheat system, helped to increase soil microbial population as compared to fallow. More pronounced effects were observed with residue incorporation. Residue incorporation not only increases the microbial population but also results in more $\mathrm{CO}_{2}$ evolution and dehydrogenase activity in the uppermost 0 $15 \mathrm{~cm}$ of the soil. The increased microbial activity later helps in the mineralization and immobilization of nutrients, especially $\mathrm{N}$ and $\mathrm{P}$. Previously it was reported that addition of a legume in the rice-wheat system improves soil microbial biomass and activity (IARI, 1995). Green manuring improves the physical condition of the soil when mungbean biomass is incorporated in a rice-wheat-mungbean sequence (IARI, 1995).

Low soil fertility due to organic matter depletion severely affects crop production in Bangladesh (BARC, 2012). Organic matter contributes to soil fertility and productivity through its positive effect on different soil properties. Bangladesh agriculture has moved to a stage 
in which intensive cropping and higher yields per unit area are being increasingly sought. Higher crop yields remove higher quantities of nutrients from the soil. Because of intensive cropping and monoculture of modern varieties of rice, soil fertility declines and deficiencies of nutrients arise. Naturally, all soils contain all nutrients, but their reserves are not similar and unlimited. Nutrients may be depleted because of increased cropping intensity, use of smaller amounts of organic matter, high rainfall, soil erosion, and leaching. Low organic matter content appears to be associated with rapid decomposition of organic matter on the one hand, and very little addition of organic matter on the other. The country's sub-tropical climate and frequent tillage operations for increasing cropping intensity have resulted in enhanced rates of organic matter decomposition. Rijpma and Jahiruddin (2004) reported that the nutrient balance for $\mathrm{N}$ is moderately negative, for $\mathrm{P}$ is slightly negative, and for K is highly negative in this country's soils.

This undesirable consequence may be overcome by the addition of adequate amounts of organic matter to the soil from different sources such as leaf manure and green manure, compost, cowdung, oilcake, crop residues, and other organic wastes. Inclusion of leguminous green manure in the rice-wheat cropping system enhanced and sustained rice production in the wet season (IRRI, 1988). The addition of green manure alone can help to make soil fertile, but the combined application of green manure and nitrogenous fertilizer increases the yield of rice further, as well as the availability of NPK in the soil and the nutrient uptake capacity of rice plants (Tiwari et al., 1980). If green manuring is applied along with nitrogenous fertilizer, it helps to release nutrient elements slowly during the whole period of crop growth (Singh et al., 1990). In another study it was found that addition of green manure in combination with chemical fertilizers produced a higheryield than did a single application of chemical fertilizer alone (Aktar et al., 1993). Thus, an integrated use of green manure and chemical fertilizer is needed to supply the nutrients needed to obtain a higher rice yield. Fertilizer is a major input in modern farming since about $50 \%$ of the world's crop production depends on this input (Pradhan, 1992). However, sustainable higher crop production cannot be maintained by using inorganic fertilizers alone, nor it is possible to obtain higher crop yields by using only organic manure. In this context, integrated plant nutrient management with a combined use of inorganic and organic sources of nutrients can be a good approach to combat nutrient depletion and promote sustainable crop production. Therefore, the aim of our work was to develop a package of organic and inorganic components and to understand the effect of those components on the growth and yield of transplanted aman (T. aman) rice, as well as to study their influence on post-harvest soil nutrient status.

\section{Materials and Methods}

\section{Site and Soil Conditions}

The experiment was carried out at the Regional Wheat Research Station of the Bangladesh Agricultural Research Institute, Shyampur, Rajshahi, Bangladesh (28027/N latitude and 92058/E longitude) for two consecutive years. The experimental land is high in topography and characterized by a clay loam texture having $\mathrm{pH} 8.10$, OM $1.08 \%$, total $\mathrm{N} 0.06 \%, \mathrm{P}$ $9.57 \mathrm{mg} \mathrm{kg}^{-1}, \mathrm{~K} 0.217 \mathrm{mg} 100 \mathrm{~g}^{-1}$, S $21.89 \mathrm{mg} \mathrm{kg}^{-1}$, and $\mathrm{Zn} 0.460 \mathrm{mg} \mathrm{kg}^{-1}$.

\section{Experimental Treatments and Design}

The treatments included in the study were five green manuring crops (Crotalaria juncea, Sesbania rostrata, Sesbania aculeata, Vigna radiata, and Phaseolus mungo), seasonal fallow, and four levels of nitrogen ( 0 , 40,80 , and $120 \mathrm{~kg} \mathrm{ha}^{-1}$ ). The experiment was laid out in a split-plot design assigning green manuring crops in the main plot and nitrogen doses in the sub-plots with three replications. The unit size of the sub-plots was 4 $\mathrm{m} \times 3 \mathrm{~m}$ and that of main plot was $48 \mathrm{~m}^{2}$. The variety BRRI dhan39 (Oryza sativa L.) of T. aman rice was grown as the test variety.

\section{Cultural Practices}

Seeds of green manuring crops were sown on 10 May in the first year and on 8 May in the second year. They were fertilized with $\mathrm{N}, \mathrm{P}, \mathrm{K}$, and $\mathrm{S}$ at 10, 20, 30, and 15 $\mathrm{kg} \mathrm{ha}^{-1}$, respectively, in the form of urea, triple super phosphate (TSP), muriate of potash (MOP), and gypsum. The seedlings of transplanted aman rice were raised in a wet nursery-bed. Sprouted rice seeds were sown on 24 June during the first year and on 22 June during the second year in a well-prepared nursery-bed. Irrigation and insect pest management were performed as needed. The experimental plots were irrigated, ploughed, and cross-ploughed, followed by laddering to create a good puddled condition. At the final stage of land preparation, the experimental plots were fertilized with $\mathrm{P}, \mathrm{K}, \mathrm{S}$ and $\mathrm{Zn}$ at a rate of $18,40,12$, and $2 \mathrm{~kg} \mathrm{ha}^{-1}$, respectively, in the form of TSP, MOP, gypsum, and zinc sulfate. Different doses of nitrogen $(0,40,80$, and $120 \mathrm{~kg} \mathrm{~N}$ $\mathrm{ha}^{-1}$ ) were applied to the plots in the form of urea in three equal installments as per treatment specifications at 20, 35, and 50 days after transplanting (DAT). Green manuring crops were incorporated at 55 days after sowing. Twenty fiveday-old healthy seedlings were uprooted carefully from the seed bed and were transplanted at the rate of three seedlings hill ${ }^{-1}$ in the unit plots on 19 July in the first year and 17 July in the second year, respectively, with a spacing of $20 \mathrm{~cm} \times 15 \mathrm{~cm}$. 


\section{Measurement of Growth Parameters for T. Aman Rice}

Number of tillers hill ${ }^{-1}$, leaf area, and dry matter yield of transplanted aman rice were recorded at the flowering and grain filling stages. Plant height and number of tillers hill ${ }^{-1}$ were recorded from five randomly selected hills in each plot, excluding border hills. Leaf area, leaf dry weight, and total dry weight were recorded from another set of five hills, excluding border hills, randomly selected for destructive sampling in each plot. Each time, destructive samples were uprooted and washed with water. The leaf blades were separated from the leaf sheath, and leaf area was measured by a leaf area meter. After measurement of leaf area, the plant samples were dried in an electric oven at $65^{\circ} \mathrm{C}$ for $72 \mathrm{~h}$ until they reached a constant weight, and then their dry weights were recorded. Leaf area index (LAI), crop growth rate (CGR), net assimilation rate (NAR), relative leaf growth rate (RLGR), specific leaf area (SLA) and leaf weight ratio (LWR) were calculated following the standard formulae (Radford, 1967; Hunt, 1978).

\section{Measurements of Yield and Yield Components of T. Aman rice}

Yield and yield component parameters (plant height and number of total tillers hill ${ }^{-1}$, number of non-bearing tillers hill ${ }^{-1}$, number of effective tillers hill $^{-1}$, panicle length, number of total spikelets panicle $^{-1}$, number of unfilled spikelets panicle ${ }^{-1}$, number of grains panicle ${ }^{-1}$, and 1000-grain weight) were recorded from the set of five hills.

\section{Sampling and Chemical Analysis of Grain and Straw}

From the first and second year's experiments with transplanted aman rice, plant samples were collected for chemical analysis at harvest from five selected hills plot ${ }^{1}$ to make a composite sample. Grains and straws were separated from each composite sample and oven dried at $65^{\circ} \mathrm{C}$ for $72 \mathrm{~h}$. The samples were ground separately by a grinding machine and $12 \mathrm{~g}$ of each sample was bottled for chemical analysis. Grain and straw samples were then analyzed to determine their nitrogen content. A total of 72 grain and 72 straw samples were prepared for chemical analysis in the Soil Resources Development Institute (SRDI), Shyampur, Rajshahi, Bangladesh. Nitrogen content of the grain and straw were determined by the micro-Kjeldahl method. A $0.1 \mathrm{~g}$ grain/straw sample and a $1.1 \mathrm{~g}$ digestion tablet $\left(\mathrm{K}_{2} \mathrm{SO}_{4}: \mathrm{CuSO}_{4}+5 \mathrm{H}_{2} \mathrm{O}: \mathrm{Se}=10: 1: 0.1\right)$ were placed in a digestion tube and then $5 \mathrm{~mL}$ conc. $\mathrm{H}_{2} \mathrm{SO}_{4}$ and $2 \mathrm{~mL}$ $\mathrm{H}_{2} \mathrm{O}_{2}(30 \%)$ were added to it. The flasks were taken to the digestion chamber and the block digester was set at $350^{\circ} \mathrm{C}$ for $2 \mathrm{~h}$. After completion of digestion the tubes were cooled down for $30 \mathrm{~min}$. The digest was transferred to a volumetric flask and the volume was raised to $100 \mathrm{~mL}$ with distilled water and with $40 \%$ $\mathrm{NaOH}$ solution. The distilled $\mathrm{NH}_{3}$ was absorbed in $\mathrm{H}_{3} \mathrm{BO}_{3}$ indicator solution and titrated with $0.01 \mathrm{~N} \mathrm{H}_{2} \mathrm{SO}_{4}$. Results were expressed as percentages. Protein content of the straw and grain was determined by multiplying the nitrogen content of the straw and grain by 6.25 . Results were expressed as percentages.

Nuptake by grain and straw also were calculated by the following formula:

$$
\begin{aligned}
& N \text { - uptake by grain }\left(\mathrm{kg} \mathrm{ha}^{-1}\right) \\
& =\frac{\text { Total } N(\%) \text { in grain } \times \text { grain yield }\left(\mathrm{kg} \mathrm{ha}^{-1}\right)}{100} \\
& N-\text { uptake by straw }\left(\mathrm{kg} \mathrm{ha}^{-1}\right) \\
& =\frac{\text { Total } N(\%) \text { in straw } \times \text { straw yield }\left(\mathrm{kg} \mathrm{ha}^{-1}\right)}{100}
\end{aligned}
$$

\section{Results}

Physiological Changes of Rice Due to Green Manure and Nitrogen

\section{Relative Leaf Growth Rate (RLGR)}

The relative leaf growth rate (RLGR) of T. aman rice was influenced significantly by green manure and nitrogen addition at both the flowering stage (FS) and the grain filling stage (GFS) in both years, except during the flowering stage of year 2 (Table 1). $P$. mungo and seasonal fallow with no $\mathrm{N}$ fertilizer exhibited the highest RLGR at the flowering stage, followed by $V$. radiata and $S$. rostrata with no $\mathrm{N}$ fertilizer, and seasonal fallow with $80 \mathrm{~kg} \mathrm{ha}^{-1} \mathrm{~N}$. Green manures with all additional $\mathrm{N}$ levels showed significantly higher RLGR at GFS, except $V$. radiata with 0 and $40 \mathrm{~kg} \mathrm{ha}^{-1} \mathrm{~N}$. With little variation, a similar pattern of response was also observed in year 2. The negative value of RLGR at the flowering stage indicated that RLGR gradually decreased with an increase in the age of the plant.

\section{Specific Leaf Area (SLA)}

The combined effect of green manure and nitrogen level increase exerted a significant influence on specific leaf area (SLA) at both stages only in year 1 . The maximum SLA was noted in seasonal fallow with 120,80 , and $40 \mathrm{~kg} \mathrm{ha}^{-1} \mathrm{~N}$, S. rostrata with $80 \mathrm{~kg} \mathrm{ha}^{-1}$ $\mathrm{N}$, and $V$. radiata with 0 and $80 \mathrm{~kg} \mathrm{ha}^{-1} \mathrm{~N}$ (Table 1). However, the lowest SLA at FS was recorded in $C$. juncea with $120 \mathrm{~kg}$ and $P$. mungo with no N. $S$. aculeata with 0 and $40 \mathrm{~kg} \mathrm{ha}^{-1} \mathrm{~N}$ showed significantly higher SLA at GFS. 
Table 1. Changes in morpho-physiological traits of T.aman rice (cv. BRRI dhan39) as affected by green manures and nitrogen levels

\begin{tabular}{|c|c|c|c|c|c|c|c|c|c|c|c|c|c|}
\hline \multirow[b]{3}{*}{ Green manures } & \multirow{3}{*}{$\begin{array}{l}\text { N levels } \\
\left(\mathrm{kg} \mathrm{ha}^{-1}\right)\end{array}$} & \multicolumn{4}{|c|}{ Relative leaf growth rate $\left(\mathrm{cm}^{2} \mathrm{~cm}^{-2} \mathrm{day}^{-1}\right)$} & \multicolumn{4}{|c|}{ Specific leaf area $\left(\mathrm{cm}^{2} \mathrm{~g}^{-1}\right)$} & \multicolumn{4}{|c|}{ Leaf weight ratio $\left(\mathrm{g} \mathrm{g}^{-1}\right)$} \\
\hline & & \multicolumn{2}{|l|}{ FS } & \multicolumn{2}{|l|}{ GFS } & \multicolumn{2}{|l|}{ FS } & \multicolumn{2}{|l|}{ GFS } & \multicolumn{2}{|l|}{ FS } & \multicolumn{2}{|l|}{ GFS } \\
\hline & & Yr. 1 & Yr. 2 & Yr. 1 & Yr. 2 & Yr. 1 & Yr. 2 & Yr. 1 & Yr. 2 & Yr. 1 & Yr. 2 & Yr. 1 & Yr. 2 \\
\hline \multirow[t]{4}{*}{ Crotalaria juncea } & 0 & $0.0188 \mathrm{c}-\mathrm{g}$ & 0.0216 & $-0.0183 \mathrm{ab}$ & $-0.0189 \mathrm{~cd}$ & $285.00 \mathrm{~b}-\mathrm{f}$ & 281.47 & $238.87 \mathrm{~d}$ & 221.95 & $0.2931 \mathrm{~b}-\mathrm{e}$ & $0.2864 \mathrm{~b}-\mathrm{e}$ & $0.1448 \mathrm{bcd}$ & $0.1500 \mathrm{~d}-\mathrm{g}$ \\
\hline & 40 & $0.0171 \mathrm{~d}-\mathrm{g}$ & 0.0199 & $-0.0162 \mathrm{ab}$ & $-0.0150 \mathrm{a}-\mathrm{d}$ & $264.91 \mathrm{cef}$ & 271.29 & $238.99 \mathrm{~d}$ & 224.44 & $0.3220 \mathrm{bc}$ & $0.3044 a b c$ & $0.1580 \mathrm{ab}$ & $0.1601 \mathrm{~b}-\mathrm{e}$ \\
\hline & 80 & $0.0161 \mathrm{efg}$ & 0.0196 & $-0.0149 \mathrm{ab}$ & $-0.0120 \mathrm{a}$ & $285.42 \mathrm{~b}-\mathrm{f}$ & 282.50 & $226.16 \mathrm{~d}$ & 219.65 & $0.3012 \mathrm{bcd}$ & $0.2941 \mathrm{a}-\mathrm{e}$ & $0.1645 \mathrm{a}$ & $0.1742 \mathrm{a}$ \\
\hline & 120 & $0.0149 \mathrm{fg}$ & 0.0184 & $-0.0141 \mathrm{a}$ & $-0.0143 \mathrm{abc}$ & $237.39 \mathrm{f}$ & 256.79 & $253.40 \mathrm{~cd}$ & 229.74 & $0.3694 \mathrm{a}$ & $0.3309 \mathrm{a}$ & $0.1552 \mathrm{abc}$ & $0.1725 \mathrm{ab}$ \\
\hline \multirow[t]{4}{*}{ Sesbania aculeata } & 0 & 0.0209 cde & 0.0243 & $-0.0153 \mathrm{ab}$ & $-0.0163 a-d$ & $255.68 \mathrm{def}$ & 266.36 & $347.46 a$ & 263.55 & $0.3015 \mathrm{bcd}$ & $0.2892 \mathrm{a}-\mathrm{e}$ & $0.0961 \mathrm{k}$ & $0.1255 \mathrm{i}-1$ \\
\hline & 40 & $0.0180 \mathrm{~d}-\mathrm{g}$ & 0.0207 & $-0.0177 \mathrm{ab}$ & $-0.0164 a-d$ & $274.64 b-f$ & 276.65 & $331.90 \mathrm{ab}$ & 259.94 & $0.3086 \mathrm{bcd}$ & $0.2956 \mathrm{a}-\mathrm{e}$ & $0.1016 \mathrm{jk}$ & $0.1321 \mathrm{~h}-\mathrm{k}$ \\
\hline & 80 & $0.0170 \mathrm{~d}-\mathrm{g}$ & 0.0207 & $-0.0146 a b$ & $-0.0135 a b$ & $269.44 \mathrm{cef}$ & 274.57 & $236.53 \mathrm{~d}$ & 223.19 & $0.3033 \mathrm{bcd}$ & $0.2969 \mathrm{a}-\mathrm{e}$ & $0.1558 \mathrm{abc}$ & $0.1666 \mathrm{abc}$ \\
\hline & 120 & $0.0168 \mathrm{~d}-\mathrm{g}$ & 0.0201 & $-0.0144 a b$ & $-0.0149 a-d$ & $274.74 \mathrm{cef}$ & 277.50 & $252.38 \mathrm{~cd}$ & 229.51 & $0.3005 \mathrm{bcd}$ & $0.2988 \mathrm{a}-\mathrm{d}$ & $0.1527 \mathrm{abc}$ & $0.1661 \mathrm{abc}$ \\
\hline \multirow[t]{4}{*}{ Sesbania rostrata } & 0 & $0.0217 \mathrm{bcd}$ & 0.0245 & $-0.0164 a b$ & $-0.0143 a b c$ & $248.18 \mathrm{ef}$ & 262.07 & $258.33 \mathrm{~cd}$ & 233.19 & $0.3203 b c$ & $0.2975 \mathrm{a}-\mathrm{e}$ & $0.1211 \mathrm{e}-\mathrm{i}$ & $0.1375 \mathrm{ghi}$ \\
\hline & 40 & $0.0180 \mathrm{~d}-\mathrm{g}$ & 0.0215 & $-0.0187 \mathrm{ab}$ & $-0.0166 a-d$ & $274.74 \mathrm{cef}$ & 276.97 & $263.12 \mathrm{~cd}$ & 235.30 & $0.2956 \mathrm{bcd}$ & $0.2901 \mathrm{a}-\mathrm{e}$ & $0.1236 \mathrm{e}-\mathrm{i}$ & $0.1411 \mathrm{fgh}$ \\
\hline & 80 & $0.0144 \mathrm{~g}$ & 0.0211 & $-0.0154 a b$ & $-0.0165 a-d$ & $299.38 \mathrm{a}-\mathrm{e}$ & 289.16 & $297.67 \mathrm{bc}$ & 246.38 & $0.2774 \mathrm{c}-\mathrm{g}$ & $0.2850 \mathrm{~b}-\mathrm{e}$ & $0.1197 \mathrm{f}-\mathrm{I}$ & $0.1422 \mathrm{fgh}$ \\
\hline & 120 & $0.0180 \mathrm{~d}-\mathrm{g}$ & 0.0222 & $-0.0147 a b$ & $-0.0145 a b c$ & $252.30 \mathrm{def}$ & 265.38 & $245.45 \mathrm{~d}$ & 226.15 & $0.3353 \mathrm{ab}$ & $0.3159 \mathrm{ab}$ & $0.1478 \mathrm{abc}$ & $0.1626 a-d$ \\
\hline \multirow[t]{4}{*}{ Vigna radiata } & 0 & $0.0236 \mathrm{bc}$ & 0.0276 & $-0.0213 b$ & $-0.0201 \mathrm{~d}$ & $299.09 a-d$ & 289.95 & $240.62 \mathrm{~d}$ & 231.99 & $0.2398 \mathrm{fg}$ & $0.2584 \mathrm{de}$ & $0.1087 \mathrm{hijk}$ & $0.1227 \mathrm{jkl}$ \\
\hline & 40 & $0.0219 \mathrm{~cd}$ & 0.0255 & $-0.0213 b$ & $-0.0203 \mathrm{~d}$ & $290.12 b-f$ & 283.86 & $249.24 \mathrm{~cd}$ & 234.39 & $0.2716 \mathrm{c}-\mathrm{g}$ & $0.2836 \mathrm{~b}-\mathrm{e}$ & 0.1173 ghij & $0.1301 \mathrm{~h}-\mathrm{k}$ \\
\hline & 80 & $0.0177 \mathrm{~d}-\mathrm{g}$ & 0.0224 & $-0.0178 \mathrm{ab}$ & $-0.0180 \mathrm{bcd}$ & 288.13a-f & 283.93 & $250.81 \mathrm{~cd}$ & 232.65 & $0.2737 \mathrm{c}-\mathrm{g}$ & $0.2797 \mathrm{~b}-\mathrm{e}$ & $0.1288 \mathrm{~d}-\mathrm{g}$ & $0.1404 \mathrm{fgh}$ \\
\hline & 120 & $0.0187 \mathrm{c}-\mathrm{g}$ & 0.0222 & $-0.0167 \mathrm{ab}$ & $-0.0148 a-d$ & $283.96 b-f$ & 281.63 & $235.00 \mathrm{~d}$ & 230.03 & $0.2866 \mathrm{~b}-\mathrm{f}$ & $0.2897 a-e$ & $0.1390 \mathrm{cde}$ & $0.1534 \mathrm{c}-\mathrm{f}$ \\
\hline \multirow[t]{4}{*}{ Phaseolus mungo } & 0 & $0.0291 a^{\circ}$ & 0.0282 & $-0.0196 a b$ & $-0.0185 b c d$ & $246.59 \mathrm{f}$ & 272.07 & $243.55 \mathrm{~d}$ & 229.81 & $0.2989 \mathrm{bcd}$ & $0.2808 \mathrm{~b}-\mathrm{e}$ & $0.1165 \mathrm{~g}-\mathrm{j}$ & $0.1293 \mathrm{~h}-\mathrm{k}$ \\
\hline & 40 & $0.0195 \mathrm{c}-\mathrm{g}$ & 0.0241 & $-0.0201 \mathrm{ab}$ & $-0.0192 \mathrm{~cd}$ & $283.95 b-f$ & 292.38 & $247.51 \mathrm{~d}$ & 231.90 & $0.2805 \mathrm{c}-\mathrm{g}$ & $0.2742 \mathrm{~b}-\mathrm{e}$ & $0.1268 \mathrm{~d}-\mathrm{h}$ & $0.1362 \mathrm{~g}-\mathrm{j}$ \\
\hline & 80 & $0.0179 \mathrm{~d}-\mathrm{g}$ & 0.0223 & $-0.0172 \mathrm{ab}$ & $-0.0176 a-d$ & $274.02 \mathrm{cef}$ & 288.00 & $262.25 \mathrm{~cd}$ & 236.92 & $0.2893 \mathrm{~b}-\mathrm{f}$ & $0.2824 \mathrm{~b}-\mathrm{e}$ & $0.1285 \mathrm{~d}-\mathrm{g}$ & $0.1417 \mathrm{fgh}$ \\
\hline & 120 & $0.0186 \mathrm{c}-\mathrm{g}$ & 0.0223 & $-0.0156 \mathrm{ab}$ & $-0.0147 a b c$ & $246.15 \mathrm{ef}$ & 272.03 & $239.37 \mathrm{~d}$ & 228.37 & $0.3361 \mathrm{ab}$ & $0.3020 \mathrm{a}-\mathrm{d}$ & $0.1498 \mathrm{abc}$ & $0.1593 \mathrm{~b}-\mathrm{e}$ \\
\hline \multirow[t]{4}{*}{ Seasonal fallow } & 0 & $0.0266 \mathrm{ab}$ & 0.0286 & $-0.0160 \mathrm{ab}$ & $-0.0179 b c d$ & $251.25 \mathrm{def}$ & 266.61 & $298.56 \mathrm{bc}$ & 244.07 & $0.2678 \mathrm{~d}-\mathrm{g}$ & $0.2640 \mathrm{cde}$ & $0.0969 \mathrm{k}$ & 0.11551 \\
\hline & 40 & $0.0218 \mathrm{~cd}$ & 0.0259 & $-0.0196 a b$ & $-0.0187 \mathrm{bcd}$ & $321.23 \mathrm{abc}$ & 300.07 & $273.97 \mathrm{~cd}$ & 238.02 & $0.2327 \mathrm{~g}$ & $0.2527 \mathrm{e}$ & $0.1075 \mathrm{ijk}$ & $0.1219 \mathrm{kl}$ \\
\hline & 80 & $0.0218 \mathrm{bcd}$ & 0.0250 & $-0.0208 \mathrm{ab}$ & $-0.0201 \mathrm{~d}$ & $330.41 \mathrm{ab}$ & 303.08 & $224.65 \mathrm{~d}$ & 216.31 & $0.2445 \mathrm{efg}$ & $0.2604 \mathrm{cde}$ & 0.1391 cde & 0.1421 fgh \\
\hline & 120 & $0.0201 \mathrm{c}-\mathrm{f}$ & 0.0242 & $-0.0197 a b$ & $-0.0192 \mathrm{~cd}$ & $342.08 \mathrm{a}$ & 308.84 & $236.54 \mathrm{~d}$ & 222.13 & $0.2332 \mathrm{~g}$ & $0.2588 \mathrm{de}$ & $0.1372 \mathrm{c}-\mathrm{f}$ & $0.1469 \mathrm{efg}$ \\
\hline Level of significance & & 0.01 & NS & 0.01 & 0.01 & 0.01 & NS & 0.01 & NS & 0.01 & 0.01 & 0.01 & 0.05 \\
\hline CV $(\%)$ & & 13.66 & 15.52 & -5.8 & -14.78 & 7.66 & 7.72 & 7.56 & 10.47 & 6.67 & 7.87 & 5.3 & 5.18 \\
\hline
\end{tabular}

FS = Flowering stage GFS = Grain filling stage

\section{Leaf Weight Ratio (LWR)}

Leaf weight ratio (LWR) differed significantly due to the combined effect of green manure and nitrogen addition at both growth stages in both years. The highest LWR at FS was recorded in C. juncea, $S$. rostrata, and $P$. mungo with $120 \mathrm{~kg} \mathrm{ha}^{-1} \mathrm{~N}$ in both years (Table 1$) . S$. aculeata with all levels of additional $\mathrm{N}$ demonstrated higher LWR in year 2. The highest LWR at GFS was found in C. juncea with 40, 80, and $120 \mathrm{~kg} \mathrm{ha}^{-1} \mathrm{~N}, S$. aculeata with 80 and $120 \mathrm{~kg} \mathrm{ha}^{-1} \mathrm{~N}, S$. rostrata with 120 $\mathrm{kg} \mathrm{ha}^{-1} \mathrm{~N}$, and $P$. mungo with $120 \mathrm{~kg} \mathrm{ha}^{-1} \mathrm{~N}$ in year 1 . More or less similar responses were noted in year 2 .

\section{Leaf Area Index (LAI)}

Leaf area index (LAI) was significantly affected by the combination of green manure and $\mathrm{N}$ level in both years. The highest LAI at FS was attained by $C$. juncea, $S$. aculeata, and $S$. rostrata coupled with 80 and $120 \mathrm{~kg} \mathrm{ha}^{-1}$ $\mathrm{N}$, and $V$. radiata and $P$. mungo with $120 \mathrm{~kg} \mathrm{ha}^{-1} \mathrm{~N}$ (Table 2 ). With some variation, a similar response was noted at GFS in both years. An increase in LAI was observed at higher levels of nitrogen in all the treatment combinations, irrespective of the green manuring species used. However, the lowest LAI was recorded in seasonal fallow without any $\mathrm{N}$ fertilizer. The results also indicated that the value of LAI was relatively higher than GFS at FS in both years.

\section{Crop Growth Rate (CGR)}

Crop growth rate responded significantly to the combined effect of green manures and $\mathrm{N}$ addition (Table 2 ). The results generally showed that all green manure crops and seasonal fallow, coupled with any $\mathrm{N}$ fertilizer rate except $0 \mathrm{~kg} \mathrm{ha}^{-1}$, showed significantly higher CGR at FS in both study periods (Table 2). It seems that CGR at FS increases with higher levels of $\mathrm{N}$ in all the treatment combinations irrespective of green manure type. Similar CGR results were observed at GFS in year 2. However, CGR at GFS did not respond to the combination of green manure and $\mathrm{N}$ level in year 1 (Table 2).

\section{Net Assimilation Rate (NAR)}

Net assimilation rate (NAR) was also significantly influenced by the combined effect of green manure and $\mathrm{N}$ addition. Except for a little variation, we observed that green manure crops, particularly $S$. aculeata, $S$. rostrata, $V$. radiata, and $P$. mungo, in combination with lower N levels, increased NAR at FS in the study period (Table 2). The NAR at GFS showed variation when compared to FS. All increased N levels, along with $S$. rostrata, $V$. radiata, and seasonal fallow, resulted in higher NAR at GFS. A nonsignificant response was noted for NAR at GFS in year 2.

\section{Yield and Yield Contributing Characters}

The number of effective tillers hill ${ }^{-1}$ of T.aman rice was significantly influenced by green manure and nitrogen addition in both years (Table 3 ). All green manure crops combined with 40,80 , and $120 \mathrm{~kg} \mathrm{ha}^{-1} \mathrm{~N}$, and seasonal fallow with $120 \mathrm{~kg} \mathrm{ha}^{-1} \mathrm{~N}$, produced the highest number of effective tillers hill $^{-1}$. Seasonal fallow with zero nitrogen gave the lowest number of effective tillers hill ${ }^{-1}$ in both years (Table 3). The combination of green manure and nitrogen level had a significant effect on grains panicle ${ }^{-1}$. In both study periods, all green manure crops with different levels of additional $\mathrm{N}$ showed significantly higher numbers of grains panicle ${ }^{-1}$, except $V$. radiata and $P$. mungo with $0 \mathrm{kgN} \mathrm{ha}^{-1}$, and seasonal fallow with 0 and $40 \mathrm{~kg} \mathrm{~N} \mathrm{ha}^{-1}$ (Table 3). The combined effect of green manure and nitrogen level on 1000-grain weight was also found to be non-significant in both years (Table 3 ). 
Table 2. Changes in morpho-physiological traits of T.aman rice (cv. BRRI dhan39) as affected by green manures and nitrogen levels

\begin{tabular}{|c|c|c|c|c|c|c|c|c|c|c|c|c|c|}
\hline \multirow[b]{3}{*}{ Green manures } & \multirow{3}{*}{$\begin{array}{l}\text { N levels } \\
\left(\mathrm{kg} \mathrm{ha}^{-1}\right)\end{array}$} & \multicolumn{4}{|c|}{ Leaf area index } & \multicolumn{3}{|c|}{ Crop growth rate $\left(\mathrm{g} \mathrm{m}^{-2}\right.$ day $\left.^{-1}\right)$} & \multicolumn{3}{|c|}{ Net assimilation rate $\left(\mathrm{mg} \mathrm{cm}^{-2} \mathrm{day}^{-1}\right)$} & & \\
\hline & & \multicolumn{2}{|l|}{ FS } & \multicolumn{2}{|l|}{ GFS } & \multicolumn{2}{|l|}{ FS } & \multicolumn{2}{|l|}{ GFS } & \multicolumn{2}{|l|}{ FS } & & \\
\hline & & Yr. 1 & Yr. 2 & Yr. 1 & Yr. 2 & Yr. 1 & Yr. 2 & Yr. 1 & Yr. 2 & Yr. 1 & Yr. 2 & Yr. 1 & Yr. 2 \\
\hline \multirow[t]{4}{*}{ Crotalaria juncea } & 0 & $3.99 \mathrm{f}-\mathrm{j}$ & $4.01 \mathrm{f}-\mathrm{j}$ & $2.47 \mathrm{~g}-1$ & $2.47 \mathrm{f}-\mathrm{j}$ & $12.44 \mathrm{bc}$ & $13.60 \mathrm{c}-\mathrm{f}$ & 6.45 & $7.29 \mathrm{abc}$ & $0.3582 \mathrm{bc}$ & $0.3981 \mathrm{bcd}$ & $0.2294 \mathrm{cde}$ & 0.2514 \\
\hline & 40 & $4.68 \mathrm{~b}-\mathrm{f}$ & $4.71 \mathrm{a}-\mathrm{f}$ & $2.95 \mathrm{c}-\mathrm{g}$ & $3.05 b-f$ & $14.38 \mathrm{abc}$ & $16.03 \mathrm{a}-\mathrm{e}$ & 7.55 & $8.47 \mathrm{ab}$ & $0.3487 \mathrm{bc}$ & $0.3950 \mathrm{bcd}$ & $0.2284 \mathrm{cde}$ & 0.2449 \\
\hline & 80 & $5.09 \mathrm{abc}$ & $5.16 \mathrm{abc}$ & $3.36 \mathrm{a}-\mathrm{d}$ & $3.53 \mathrm{abc}$ & $15.56 \mathrm{abc}$ & $17.41 \mathrm{ab}$ & 8.58 & $9.17 \mathrm{a}$ & $0.3443 \mathrm{bc}$ & $0.3905 \mathrm{bcd}$ & $0.2281 \mathrm{cde}$ & 0.2344 \\
\hline & 120 & $5.46 \mathrm{a}$ & $5.54 \mathrm{a}$ & $3.76 \mathrm{a}$ & $3.82 \mathrm{a}$ & $16.25 \mathrm{abc}$ & $18.19 \mathrm{a}$ & 8.61 & $8.56 \mathrm{ab}$ & $0.3324 \mathrm{bc}$ & $0.3768 \mathrm{~cd}$ & $0.2080 \mathrm{e}$ & 0.1979 \\
\hline \multirow[t]{4}{*}{ Sesbania aculeata } & 0 & $3.75 \mathrm{~g}-\mathrm{j}$ & $3.77 \mathrm{~g}-\mathrm{j}$ & $2.37 \mathrm{i}-1$ & $2.39 \mathrm{f}-\mathrm{j}$ & $14.08 \mathrm{abc}$ & $14.20 \mathrm{~b}-\mathrm{f}$ & 6.45 & $6.77 \mathrm{bc}$ & $0.4410 \mathrm{abc}$ & $0.4503 a-d$ & $0.2415 \mathrm{~b}-\mathrm{e}$ & 0.2461 \\
\hline & 40 & $4.44 \mathrm{c}-\mathrm{g}$ & $4.48 \mathrm{~b}-\mathrm{h}$ & $2.77 \mathrm{e}-\mathrm{j}$ & $2.85 \mathrm{c}-\mathrm{g}$ & $12.73 \mathrm{bc}$ & $14.77 \mathrm{~b}-\mathrm{f}$ & 8.08 & $7.85 \mathrm{ab}$ & $0.3283 \mathrm{c}$ & $0.3846 \mathrm{bcd}$ & $0.2549 \mathrm{a}-\mathrm{e}$ & 0.2392 \\
\hline & 80 & $4.85 \mathrm{a}-\mathrm{d}$ & $4.92 \mathrm{a}-\mathrm{e}$ & $3.23 \mathrm{~b}-\mathrm{e}$ & $3.33 a-d$ & $16.56 \mathrm{ab}$ & $16.85 \mathrm{abc}$ & 8.88 & $8.58 \mathrm{ab}$ & $0.3872 \mathrm{bc}$ & $0.3991 \mathrm{bcd}$ & $0.2483 \mathrm{~b}-\mathrm{e}$ & 0.2288 \\
\hline & 120 & $5.22 \mathrm{ab}$ & $5.31 \mathrm{ab}$ & $3.53 \mathrm{ab}$ & $3.60 \mathrm{ab}$ & $17.44 a$ & $18.25 \mathrm{a}$ & 8.92 & $8.86 \mathrm{ab}$ & $0.3782 \mathrm{bc}$ & $0.3992 \mathrm{bcd}$ & $0.2261 \mathrm{de}$ & 0.2163 \\
\hline \multirow[t]{4}{*}{ Sesbania rostrata } & 0 & 3.64hij & 3.66hij & $2.20 \mathrm{klm}$ & $2.28 \mathrm{~g}-\mathrm{k}$ & $11.81 \mathrm{c}$ & $13.09 \mathrm{ef}$ & 7.55 & $7.00 \mathrm{abc}$ & $0.3823 \mathrm{bc}$ & $0.4287 \mathrm{bcd}$ & $0.3060 \mathrm{ab}$ & 0.2721 \\
\hline & 40 & $4.35 \mathrm{c}-\mathrm{h}$ & $4.40 \mathrm{c}-\mathrm{i}$ & $2.63 \mathrm{f}-\mathrm{k}$ & $2.73 \mathrm{~d}-\mathrm{i}$ & $16.07 \mathrm{abc}$ & $15.93 \mathrm{a}-\mathrm{e}$ & 8.76 & $8.52 \mathrm{ab}$ & $0.4226 \mathrm{abc}$ & $0.4245 \mathrm{bcd}$ & $0.2911 \mathrm{a}-\mathrm{d}$ & 0.2713 \\
\hline & 80 & $4.79 \mathrm{a}-\mathrm{e}$ & $4.85 \mathrm{a}-\mathrm{f}$ & $3.08 \mathrm{~b}-\mathrm{f}$ & $3.08 \mathrm{~b}-\mathrm{f}$ & $14.80 \mathrm{abc}$ & $16.24 \mathrm{a}-\mathrm{e}$ & 8.78 & $8.00 \mathrm{ab}$ & $0.3438 b c$ & $0.3915 \mathrm{bcd}$ & $0.2536 \mathrm{a}-\mathrm{e}$ & 0.2258 \\
\hline & 120 & $5.13 \mathrm{abc}$ & $5.21 \mathrm{abc}$ & $3.40 \mathrm{abc}$ & $3.45 \mathrm{a}-\mathrm{d}$ & $14.65 \mathrm{bc}$ & $16.60 \mathrm{a}-\mathrm{d}$ & 9.97 & $8.82 \mathrm{ab}$ & $0.3261 \mathrm{c}$ & $0.3759 \mathrm{~d}$ & $0.2621 \mathrm{a}-\mathrm{e}$ & 0.2258 \\
\hline \multirow[t]{4}{*}{ Vigna radiata } & 0 & $3.29 \mathrm{jk}$ & $3.40 \mathrm{jk}$ & $1.77 \mathrm{~m}$ & $1.92 \mathrm{jk}$ & $11.99 \mathrm{bc}$ & $13.03 \mathrm{ef}$ & 6.21 & $6.71 \mathrm{bc}$ & $0.4323 \mathrm{abc}$ & $0.4690 \mathrm{ab}$ & $0.3015 \mathrm{abc}$ & 0.2949 \\
\hline & 40 & $4.11 \mathrm{~d}-\mathrm{i}$ & $4.15 \mathrm{~d}-\mathrm{j}$ & $2.25 \mathrm{j}-\mathrm{m}$ & $2.40 \mathrm{f}-\mathrm{j}$ & $13.96 \mathrm{abc}$ & $14.47 \mathrm{~b}-\mathrm{f}$ & 7.45 & $6.96 a b c$ & $0.3982 \mathrm{bc}$ & $0.4210 \mathrm{bcd}$ & $0.2835 \mathrm{a}-\mathrm{d}$ & 0.2442 \\
\hline & 80 & $4.37 \mathrm{c}-\mathrm{h}$ & $4.49 \mathrm{~b}-\mathrm{h}$ & $2.70 \mathrm{e}-\mathrm{k}$ & $2.79 \mathrm{~d}-\mathrm{i}$ & $15.24 a b c$ & $15.92 \mathrm{a}-\mathrm{e}$ & 8.49 & $7.45 \mathrm{abc}$ & $0.3969 \mathrm{bc}$ & $0.4185 \mathrm{bcd}$ & $0.2767 \mathrm{a}-\mathrm{e}$ & 0.2295 \\
\hline & 120 & $4.78 \mathrm{a}-\mathrm{e}$ & $4.87 \mathrm{a}-\mathrm{e}$ & $2.92 \mathrm{c}-\mathrm{h}$ & $3.21 \mathrm{a}-\mathrm{e}$ & $14.30 \mathrm{abc}$ & 16.05 a-e & 9.00 & $8.06 \mathrm{ab}$ & $0.3427 \mathrm{bc}$ & $0.3885 \mathrm{bcd}$ & $0.2737 \mathrm{a}-\mathrm{e}$ & 0.2211 \\
\hline \multirow[t]{4}{*}{ Phaseolus mungo } & 0 & $3.37 \mathrm{ijk}$ & $3.50 \mathrm{jk}$ & $1.991 \mathrm{~m}$ & $2.06 \mathrm{ijk}$ & $12.47 \mathrm{bc}$ & $13.13 \mathrm{ef}$ & 7.12 & $6.90 \mathrm{bc}$ & $0.4573 \mathrm{ab}$ & $0.4611 \mathrm{abc}$ & $0.3077 \mathrm{ab}$ & 0.2865 \\
\hline & 40 & $4.07 \mathrm{~d}-\mathrm{i}$ & $4.23 \mathrm{~d}-\mathrm{j}$ & $2.43 \mathrm{~g}-1$ & $2.53 \mathrm{e}-\mathrm{j}$ & $14.20 \mathrm{abc}$ & $14.47 \mathrm{~b}-\mathrm{f}$ & 7.30 & $7.54 a b c$ & $0.4029 \mathrm{bc}$ & $0.4087 \mathrm{bcd}$ & $0.2566 \mathrm{a}-\mathrm{e}$ & 0.2532 \\
\hline & 80 & $4.43 \mathrm{c}-\mathrm{g}$ & $4.62 \mathrm{~b}-\mathrm{g}$ & $2.84 \mathrm{~d}-\mathrm{i}$ & $2.90 \mathrm{~b}-\mathrm{g}$ & $14.18 \mathrm{abc}$ & $15.55 \mathrm{a}-\mathrm{e}$ & 7.76 & $7.51 \mathrm{abc}$ & $0.3653 \mathrm{bc}$ & $0.3973 \mathrm{bcd}$ & $0.2393 \mathrm{~b}-\mathrm{e}$ & 0.2227 \\
\hline & 120 & $4.80 \mathrm{a}-\mathrm{e}$ & $4.99 \mathrm{a}-\mathrm{d}$ & $3.21 \mathrm{~b}-\mathrm{e}$ & $3.35 \mathrm{a}-\mathrm{d}$ & $15.39 \mathrm{abc}$ & $16.85 \mathrm{abc}$ & 8.19 & $8.29 \mathrm{ab}$ & $0.3681 \mathrm{bc}$ & $0.3979 \mathrm{bcd}$ & $0.2264 \mathrm{cde}$ & 0.2182 \\
\hline \multirow[t]{4}{*}{ Seasonal fallow } & 0 & $2.68 \mathrm{k}$ & $2.772 \mathrm{k}$ & $1.74 \mathrm{~m}$ & $1.67 \mathrm{k}$ & $11.93 \mathrm{c}$ & $11.84 \mathrm{f}$ & 6.46 & $5.53 \mathrm{c}$ & $0.5402 \mathrm{a}$ & $0.5261 \mathrm{a}$ & $0.3279 a$ & 0.2844 \\
\hline & 40 & $3.48 \mathrm{ij}$ & $3.570 \mathrm{ij}$ & $2.061 \mathrm{~m}$ & $2.08 \mathrm{~h}-\mathrm{k}$ & $13.20 \mathrm{abc}$ & $13.52 \mathrm{def}$ & 7.14 & $6.93 \mathrm{bc}$ & $0.4451 \mathrm{abc}$ & $0.4583 \mathrm{a}-\mathrm{d}$ & $0.2985 \mathrm{a}-\mathrm{d}$ & 0.2847 \\
\hline & 80 & $4.02 \mathrm{e}-\mathrm{j}$ & $4.079 \mathrm{e}-\mathrm{j}$ & $2.40 \mathrm{~h}-1$ & $2.43 \mathrm{f}-\mathrm{j}$ & $13.55 \mathrm{abc}$ & $14.45 \mathrm{~b}-\mathrm{f}$ & 7.20 & $7.63 \mathrm{abc}$ & $0.3955 \mathrm{bc}$ & $0.4257 \mathrm{bcd}$ & $0.2557 \mathrm{a}-\mathrm{e}$ & 0.2653 \\
\hline & 120 & $4.39 \mathrm{c}-\mathrm{h}$ & $4.51 \mathrm{~b}-\mathrm{g}$ & $2.76 \mathrm{e}-\mathrm{j}$ & $2.81 \mathrm{c}-\mathrm{h}$ & 14.64abc & $15.72 \mathrm{a}-\mathrm{e}$ & 8.36 & $7.73 \mathrm{ab}$ & $0.3865 \mathrm{bc}$ & $0.4170 \mathrm{bcd}$ & $0.2602 \mathrm{a}-\mathrm{e}$ & 0.2344 \\
\hline Level of significance & & 0.01 & $0.01^{\circ}$ & 0.01 & 0.01 & 0.01 & 0.01 & NS & 0.01 & 0.01 & 0.05 & 0.01 & NS \\
\hline CV (\%) & & 7.01 & 7.52 & 7.78 & 10.31 & 12.09 & 8.12 & 14.3 & 10.85 & 3.02 & 10.12 & 10.83 & 9.33 \\
\hline
\end{tabular}

$\mathrm{FS}=$ Flowering stage GFS $=$ Grain filling stage

Table 3. Influence of green manuring crops and nitrogen levels on yield and yield contributing traits of T.aman rice (cv. BRRI dhan39)

\begin{tabular}{|c|c|c|c|c|c|c|c|c|c|c|c|c|c|}
\hline \multirow[b]{2}{*}{ Green manures } & \multirow{2}{*}{$\begin{array}{l}\text { Nitrogen } \\
\text { levels } \\
\left(\mathrm{kg} \mathrm{ha}^{-1}\right)\end{array}$} & \multicolumn{2}{|c|}{$\begin{array}{l}\text { No. of effective } \\
\text { tillers hill }\end{array}$} & \multicolumn{2}{|c|}{$\begin{array}{l}\text { No. of grains } \\
\text { panicle }^{-1}\end{array}$} & \multicolumn{2}{|c|}{$\begin{array}{l}\text { Weight of } 1000 \\
\text { grains }(\mathrm{g})\end{array}$} & \multicolumn{2}{|c|}{$\begin{array}{l}\text { Grain yield } \\
\left(\mathrm{t} \mathrm{ha}^{-1}\right)\end{array}$} & \multicolumn{2}{|c|}{$\begin{array}{l}\text { Straw yield } \\
\left(\mathrm{t} \mathrm{ha}^{-1}\right)\end{array}$} & \multicolumn{2}{|c|}{$\begin{array}{l}\text { Harvest index } \\
\text { (\%) }\end{array}$} \\
\hline & & Yr. 1 & Yr. 2 & Yr. 1 & Yr. 2 & Yr. 1 & Yr. 2 & Yr. 1 & Yr. 2 & Yr. 1 & Yr. 2 & Yr. 1 & Yr. 2 \\
\hline \multirow{4}{*}{ Crotalaria juncea } & 0 & $7.79 \mathrm{bg}$ & $8.15 \mathrm{a}-\mathrm{e}$ & $115.31 \mathrm{ab}$ & $114.19 \mathrm{abc}$ & 22.24 & 22.97 & $3.88 \mathrm{bc}$ & $3.93 \mathrm{c}$ & $4.86 \mathrm{c}-\mathrm{g}$ & $4.93 \mathrm{~b}-\mathrm{h}$ & 44.39 & 44.41 \\
\hline & 40 & $8.89 \mathrm{abc}$ & $9.45 \mathrm{a}-\mathrm{d}$ & $128.86 a$ & 129.13abc & 22.98 & 23.84 & $5.01 \mathrm{a}$ & $5.06 \mathrm{ab}$ & $5.96 \mathrm{abc}$ & $6.00 \mathrm{a}-\mathrm{g}$ & 45.66 & 45.76 \\
\hline & 80 & $9.26 \mathrm{a}$ & $9.84 \mathrm{ab}$ & $129.37 \mathrm{a}$ & $129.93 \mathrm{ab}$ & 23.22 & 24.31 & $5.11 \mathrm{a}$ & $5.18 \mathrm{a}$ & $6.46 \mathrm{a}$ & $6.48 \mathrm{ab}$ & 44.17 & 44.41 \\
\hline & 120 & $9.44 \mathrm{a}$ & $10.10 \mathrm{a}$ & $131.49 \mathrm{a}$ & $131.33 \mathrm{a}$ & 22.88 & 24.19 & $5.16 \mathrm{a}$ & $5.22 \mathrm{a}$ & $6.57 \mathrm{a}$ & $6.70 \mathrm{a}$ & 43.98 & 43.78 \\
\hline \multirow[t]{4}{*}{ Sesbania aculeata } & 0 & $7.53 \mathrm{dg}$ & 7.92a-e & $112.13 \mathrm{ab}$ & $111.01 \mathrm{abc}$ & 22.52 & 23.01 & $3.66 \mathrm{~cd}$ & $3.75 \mathrm{~cd}$ & $4.68 \mathrm{~b}-\mathrm{g}$ & $4.77 \mathrm{~d}-\mathrm{h}$ & 43.91 & 44.02 \\
\hline & 40 & $8.70 \mathrm{a}-\mathrm{e}$ & $9.31 \mathrm{a}-\mathrm{d}$ & $125.26 \mathrm{ab}$ & $125.10 \mathrm{abc}$ & 22.75 & 23.60 & $4.87 \mathrm{a}$ & $4.91 \mathrm{ab}$ & $5.80 \mathrm{a}-\mathrm{d}$ & $5.85 \mathrm{a}-\mathrm{g}$ & 45.63 & 45.62 \\
\hline & 80 & $9.09 \mathrm{ab}$ & $9.67 a-d$ & $126.85 \mathrm{ab}$ & $127.00 \mathrm{abc}$ & 22.86 & 24.10 & $4.92 \mathrm{a}$ & $5.01 \mathrm{ab}$ & $6.18 \mathrm{ab}$ & $6.26 \mathrm{a}-\mathrm{d}$ & 44.39 & 44.43 \\
\hline & 120 & $9.29 \mathrm{a}$ & $9.91 \mathrm{a}$ & $130.08 \mathrm{a}$ & 129.19abc & 22.71 & 24.17 & $4.95 \mathrm{a}$ & $5.05 \mathrm{ab}$ & $6.33 \mathrm{a}$ & $6.50 \mathrm{ab}$ & 43.88 & 43.73 \\
\hline \multirow[t]{4}{*}{ Sesbania rostrata } & 0 & 7.49efg & $7.82 \mathrm{a}-\mathrm{e}$ & $109.02 \mathrm{ab}$ & $108.36 \mathrm{a}-\mathrm{d}$ & 22.32 & 22.82 & $3.66 \mathrm{~cd}$ & $3.67 \mathrm{~cd}$ & $4.57 \mathrm{efg}$ & $4.66 \mathrm{eh}$ & 44.53 & 44.04 \\
\hline & 40 & $8.59 \mathrm{a}-\mathrm{e}$ & $9.20 \mathrm{a}-\mathrm{d}$ & $124.20 \mathrm{ab}$ & $123.46 \mathrm{abc}$ & 22.59 & 23.60 & $4.71 \mathrm{a}$ & $4.85 \mathrm{ab}$ & $5.75 \mathrm{a}-\mathrm{d}$ & $5.78 \mathrm{a}-\mathrm{g}$ & 45.00 & 45.62 \\
\hline & 80 & $8.94 a b c$ & $9.61 \mathrm{a}-\mathrm{d}$ & $123.48 \mathrm{ab}$ & $124.20 \mathrm{abc}$ & 22.73 & 23.78 & $4.86 a$ & $4.91 \mathrm{ab}$ & $6.07 \mathrm{ab}$ & $6.15 \mathrm{a}-\mathrm{f}$ & 44.47 & 44.38 \\
\hline & 120 & $9.18 \mathrm{a}$ & $9.80 \mathrm{ab}$ & $127.46 \mathrm{ab}$ & $127.31 \mathrm{abc}$ & 22.50 & 24.05 & $4.93 a$ & $4.99 \mathrm{ab}$ & $6.31 \mathrm{ab}$ & $6.42 \mathrm{abc}$ & 43.84 & 43.73 \\
\hline \multirow[t]{4}{*}{ Vigna radiata } & 0 & $7.08 \mathrm{fgh}$ & $7.42 \mathrm{cde}$ & $104.27 b c$ & $103.81 \mathrm{~cd}$ & 22.12 & 22.75 & $3.16 \mathrm{~b}$ & $3.30 \mathrm{~d}$ & $4.41 \mathrm{fg}$ & $4.46 \mathrm{gh}$ & 41.70 & 42.54 \\
\hline & 40 & $8.20 \mathrm{a}-\mathrm{f}$ & $9.08 \mathrm{a}-\mathrm{d}$ & $113.46 \mathrm{ab}$ & $113.07 \mathrm{abc}$ & 22.40 & 23.74 & $4.47 \mathrm{ab}$ & $4.50 \mathrm{~b}$ & $5.50 \mathrm{a}-\mathrm{f}$ & $5.54 \mathrm{a}-\mathrm{g}$ & 44.81 & 44.86 \\
\hline & 80 & $8.72 \mathrm{a}-\mathrm{e}$ & $9.28 \mathrm{a}-\mathrm{d}$ & $119.47 \mathrm{ab}$ & $119.41 \mathrm{abc}$ & 22.53 & 23.61 & $4.54 \mathrm{a}$ & $4.63 \mathrm{ab}$ & $5.88 \mathrm{abc}$ & $5.95 \mathrm{a}-\mathrm{g}$ & 43.59 & 43.80 \\
\hline & 120 & $8.99 \mathrm{abc}$ & $9.62 \mathrm{a}-\mathrm{d}$ & $122.68 \mathrm{ab}$ & $122.69 a b c$ & 22.67 & 23.83 & $4.80 \mathrm{a}$ & $4.91 \mathrm{ab}$ & $6.12 \mathrm{ab}$ & $6.23 \mathrm{a}-\mathrm{e}$ & 43.90 & 44.11 \\
\hline \multirow[t]{4}{*}{ Phaseolus mungo } & 0 & $7.13 \mathrm{fgh}$ & $7.54 \mathrm{~b}-\mathrm{e}$ & $104.96 \mathrm{bc}$ & $105.24 \mathrm{bcd}$ & 22.25 & 22.87 & $3.45 \mathrm{~cd}$ & $3.49 \mathrm{~cd}$ & 4.51efg & $4.57 \mathrm{f}-\mathrm{h}$ & 43.33 & 43.34 \\
\hline & 40 & $8.31 \mathrm{a}-\mathrm{f}$ & $9.14 a-d$ & $115.06 \mathrm{ab}$ & $116.35 \mathrm{abc}$ & 22.50 & 23.84 & $4.57 \mathrm{a}$ & $4.61 \mathrm{ab}$ & $5.55 \mathrm{a}-\mathrm{e}$ & $5.62 \mathrm{a}-\mathrm{g}$ & 45.19 & 45.06 \\
\hline & 80 & $8.85 a-d$ & $9.45 \mathrm{a}-\mathrm{d}$ & $120.25 \mathrm{ab}$ & $121.19 \mathrm{abc}$ & 22.81 & 23.94 & $4.70 \mathrm{a}$ & $4.70 \mathrm{ab}$ & $5.83 \mathrm{abc}$ & $5.98 \mathrm{a}-\mathrm{g}$ & 44.68 & 44.04 \\
\hline & 120 & $9.03 \mathrm{ab}$ & $9.70 a b c$ & 121.48ab & $123.18 \mathrm{abc}$ & 22.89 & 23.95 & $4.87 \mathrm{a}$ & $4.89 \mathrm{ab}$ & $6.21 \mathrm{ab}$ & $6.33 \mathrm{a}-\mathrm{d}$ & 43.92 & 43.60 \\
\hline \multirow[t]{4}{*}{ Seasonal fallow } & 0 & $5.97 \mathrm{~h}$ & $6.29 \mathrm{e}$ & $85.23 \mathrm{c}$ & $85.04 \mathrm{~d}$ & 21.61 & 22.28 & $2.23 \mathrm{e}$ & $2.31 \mathrm{e}$ & $2.92 \mathrm{~h}$ & $3.78 \mathrm{~h}$ & 43.32 & 37.91 \\
\hline & 40 & $6.86 \mathrm{gh}$ & $7.33 \mathrm{de}$ & $104.12 \mathrm{bc}$ & $105.03 \mathrm{bcd}$ & 22.15 & 22.95 & $3.46 \mathrm{~cd}$ & $3.39 \mathrm{~cd}$ & $4.26 \mathrm{~g}$ & $4.84 \mathrm{c}-\mathrm{h}$ & 44.85 & 41.21 \\
\hline & 80 & $7.65 \mathrm{e}-\mathrm{g}$ & $8.14 a-e$ & $111.91 \mathrm{ab}$ & $112.28 \mathrm{abc}$ & 22.45 & 23.36 & $4.55 \mathrm{a}$ & $4.59 \mathrm{ab}$ & $5.15 \mathrm{~b}-\mathrm{g}$ & $5.26 \mathrm{a}-\mathrm{h}$ & 46.90 & 46.58 \\
\hline & 120 & $8.35 \mathrm{a}-\mathrm{f}$ & $8.96 \mathrm{a}-\mathrm{d}$ & $118.74 \mathrm{ab}$ & $119.59 \mathrm{abc}$ & 22.51 & 23.51 & $4.67 \mathrm{a}$ & $4.62 \mathrm{ab}$ & $5.78 \mathrm{a}-\mathrm{d}$ & $5.89 \mathrm{a}-\mathrm{g}$ & 44.68 & 44.00 \\
\hline Level of significance & & 0.01 & 0.01 & 0.01 & 0.05 & NS & NS & 0.01 & 0.01 & 0.01 & 0.01 & NS & NS \\
\hline CV $(\%)$ & & 7.61 & 10.05 & 5.4 & 10.94 & 8.1 & 6.94 & 6.13 & 5.35 & 5.85 & 10.75 & 9.84 & 7.94 \\
\hline
\end{tabular}

The combined effect of green manure and $\mathrm{N}$ addition had a significant influence on grain yield (Table 3). It was observed that all green manures $(C$. juncea, $S$. aculeata, $S$. rostrata, $P$. mungo, and $V$. radiata) in combination with 120,80 , and $40 \mathrm{~kg} \mathrm{~N}$ $\mathrm{ha}^{-1}$, and seasonal fallow in combination with $80 \mathrm{~kg} \mathrm{~N}$ $\mathrm{ha}^{-1}$, produced significantly and identically higher grain yields. The highest grain yield of $5752 \mathrm{~kg} \mathrm{ha}^{-1}$ was observed in the plot treated with a $75 \%$ recommended dose of nitrogen (RDN) and green manure incorporated at 50 DAS (Islam et al., 2014). All green manure, and seasonal fallow with $0 \mathrm{~kg} \mathrm{~N}$, resulted the lowest grain yield. The combination of green manure and nitrogen level had a significant effect on straw yield. In both study periods, all green manure crops with 40,80, and $120 \mathrm{~kg} \mathrm{ha}^{-1} \mathrm{~N}$, and seasonal fallow with $120 \mathrm{~kg} \mathrm{ha}^{-1} \mathrm{~N}$, showed significantly higher straw yield, whereas green manure and seasonal fallow with lower $\mathrm{N}$ produced the lowest straw yield (Table 3). The harvest index (HI) of T.aman rice was not significantly influenced by the application of green manure in year 1 (Table 3 ). However, harvest index in year 2 was higher due to green manuring over seasonal fallow. Different levels of added $\mathrm{N}$ in combination with green manure did not exert significant influence on the HI of T.aman rice (Table 3). 
Changes in Nitrogen and Protein Content in Grain and Straw

\section{$N$ Content in Grain and Straw}

Nitrogen content of grain and straw of T.aman rice varied significantly due to the application of green manure and $\mathrm{N}$ fertilizer (Table 4). The $\mathrm{N}$ content in rice grain ranged from 1.127 to $1.304 \%$ and in rice straw from 0.382 to $0.528 \%$ during year 1 (Table 4). The highest $\mathrm{N}$ content in both rice grain $(1.304 \%)$ and straw $(0.528 \%)$ was observed using the treatment $C$. juncea with $120 \mathrm{~kg} \mathrm{ha}^{-1} \mathrm{~N}$, and the lowest value was noted in seasonal fallow with no nitrogen application. During second year, $\mathrm{N}$ content in grain was non-responsive to all the treatments, and $\mathrm{N}$ content in straw exhibited a more or less similar trend.

\section{Protein Content in Grain and Straw}

Protein content in the grain and straw was significantly influenced by the combined effect of green manure and increased nitrogen in year 1 (Table 4). Protein content increased in the grain and straw with higher levels of nitrogen in all the treatment combinations irrespective of the green manuring species used. Similar protein content in the grain and straw was observed in year 2 from the combined effect of green manure and increased nitrogen as in the previous year (Table 4).

\section{Changes in Nitrogen Uptake by Grain and Straw}

Nitrogen uptake by rice grain ranged from 25.13 to $67.29 \mathrm{~kg} \mathrm{ha}^{-1}$ in year 1(Table 5). The maximum $\mathrm{N}$ uptake $\left(67.29 \mathrm{~kg} \mathrm{ha}^{-1}\right)$ by rice grain was observed in $C$. juncea with $120 \mathrm{~kg} \mathrm{~N} \mathrm{ha}^{-1}$. The minimum $\mathrm{N}$ uptake of $25.13 \mathrm{~kg} \mathrm{ha}^{-1}$ was observed in seasonal fallow with no nitrogen application. In the case of rice straw during year $1, \mathrm{~N}$ uptake ranged from 11.16 to $34.68 \mathrm{~kg} \mathrm{ha}^{-1}$. The maximum $\mathrm{N}$ uptake by straw $\left(34.68 \mathrm{~kg} \mathrm{ha}^{-1}\right)$ was observed in C. juncea with $120 \mathrm{~kg} \mathrm{~N} \mathrm{ha}^{-1}$ (Table 5). The minimum $\mathrm{N}$ uptake by rice straw $\left(11.16 \mathrm{~kg} \mathrm{ha}^{-1}\right)$ was recorded in seasonal fallow with no nitrogen application. The results reveal that $\mathrm{N}$ uptake in rice grain was higher than in straw.

\section{Changes in Soil Properties After Harvest of $T$. Aman Rice}

Soil $\mathrm{pH}$ was not significantly influenced by the incorporation of green manure and additional nitrogen after harvest in either year (Table 6). With a few exceptions, a numerically increasing trend in soil $\mathrm{pH}$ was observed with higher levels of nitrogen in all the treatment combinations, irrespective of green manures, in both years. The combined effect of green manure and nitrogen was significant for total $\mathrm{N}$ and exchangeable $\mathrm{K}$ in both years and for soil organic matter in year 2.An increase in post-harvest soil nitrogen content was observed with the application of higher levels of nitrogen in combination with green manures in both years (Table 6). $C$. juncea combined with higher doses of nitrogen gave higher $\mathrm{K}$ availability in the soil, and the seasonal fallow with $40 \mathrm{~kg} \mathrm{~N} \mathrm{ha}^{-1}$ gave lower $\mathrm{K}$ availability in the soil in year 1. A similar trend in soil $\mathrm{K}$ status was observed with the combined treatment in year 2. C. juncea combined with zero nitrogen gave higher organic matter content in the soil, and seasonal fallow with $120 \mathrm{~kg} \mathrm{~N} \mathrm{ha}^{-1}$ gave the lowest organic matter content in the soil (Table 6).

Table 4. Influence of green manuring crops and nitrogen levels on $\mathrm{N}$ and Protein content in rice grain and straw of T.aman rice (cv. BRRI dhan39)

\begin{tabular}{|c|c|c|c|c|c|c|c|c|c|}
\hline & \multirow{2}{*}{$\begin{array}{l}\text { Nitrogen levels } \\
\left(\mathrm{kg} \mathrm{ha}^{-1}\right)\end{array}$} & \multicolumn{2}{|c|}{$\mathrm{N}$ content in grain $(\%)$} & \multicolumn{2}{|c|}{$\mathrm{N}$ content in straw $(\%)$} & \multicolumn{2}{|c|}{ Protein content in grain $(\%)$} & \multicolumn{2}{|c|}{ Protein content in straw (\%) } \\
\hline & & Yr. 1 & Yr. 2 & Yr. 1 & Yr. 2 & Yr. 1 & Yr. 2 & Yr. 1 & Yr. 2 \\
\hline \multirow[t]{4}{*}{ Crotalaria juncea } & 0 & $1.217 \mathrm{abc}$ & 1.235 & $0.433 a b c$ & $0.442 a-d$ & $7.61 \mathrm{abc}$ & 7.72 & $2.70 \mathrm{abc}$ & $2.76 a-d$ \\
\hline & 40 & $1.254 \mathrm{abc}$ & 1.297 & $0.451 \mathrm{abc}$ & $0.485 \mathrm{a}-\mathrm{d}$ & $7.83 \mathrm{abc}$ & 8.11 & $2.82 \mathrm{abc}$ & $3.03 \mathrm{a}-\mathrm{d}$ \\
\hline & 80 & $1.284 \mathrm{ab}$ & 1.320 & $0.509 \mathrm{ab}$ & $0.527 \mathrm{ab}$ & $8.02 \mathrm{ab}$ & 8.25 & $3.18 \mathrm{ab}$ & $3.29 \mathrm{ab}$ \\
\hline & 120 & $1.304 \mathrm{a}$ & 1.330 & $0.528 \mathrm{a}$ & $0.536 a$ & $8.15 \mathrm{a}$ & 8.31 & $3.30 \mathrm{a}$ & $3.35 \mathrm{a}$ \\
\hline \multirow[t]{4}{*}{ Sesbania aculeata } & 0 & $1.197 a b c$ & 1.235 & $0.423 a b c$ & $0.442 \mathrm{a}-\mathrm{d}$ & 7.48abc & 7.72 & $2.64 a b c$ & $2.76 a-d$ \\
\hline & 40 & $1.243 \mathrm{abc}$ & 1.286 & $0.432 \mathrm{abc}$ & $0.468 \mathrm{a}-\mathrm{d}$ & 7.77abc & 8.03 & $2.70 \mathrm{abc}$ & $2.93 a-d$ \\
\hline & 80 & $1.264 \mathrm{abc}$ & 1.289 & $0.460 \mathrm{abc}$ & $0.478 \mathrm{a}-\mathrm{d}$ & $7.90 \mathrm{abc}$ & 8.06 & $2.88 \mathrm{abc}$ & $2.98 \mathrm{a}-\mathrm{d}$ \\
\hline & 120 & $1.284 \mathrm{ab}$ & 1.299 & $0.509 \mathrm{ab}$ & $0.527 \mathrm{ab}$ & $8.02 \mathrm{ab}$ & 8.12 & $3.18 \mathrm{ab}$ & $3.29 \mathrm{ab}$ \\
\hline \multirow[t]{4}{*}{ Sesbania rostrata } & 0 & $1.197 \mathrm{abc}$ & 1.224 & $0.402 b c$ & $0.431 \mathrm{a}-\mathrm{d}$ & 7.48abc & 7.65 & $2.52 b c$ & $2.69 \mathrm{a}-\mathrm{d}$ \\
\hline & 40 & $1.223 \mathrm{abc}$ & 1.266 & $0.432 \mathrm{abc}$ & $0.445 \mathrm{a}-\mathrm{d}$ & 7.64abc & 7.91 & $2.70 \mathrm{abc}$ & $2.78 \mathrm{a}-\mathrm{d}$ \\
\hline & 80 & $1.243 \mathrm{abc}$ & 1.265 & $0.490 \mathrm{abc}$ & $0.507 a-d$ & $7.77 \mathrm{abc}$ & 7.91 & $3.06 \mathrm{abc}$ & $3.17 \mathrm{abc}$ \\
\hline & 120 & $1.258 \mathrm{abc}$ & 1.289 & $0.509 \mathrm{ab}$ & $0.517 \mathrm{abc}$ & $7.86 \mathrm{abc}$ & 8.06 & $3.18 \mathrm{ab}$ & $3.23 \mathrm{abc}$ \\
\hline \multirow[t]{4}{*}{ Vigna radiata } & 0 & $1.141 \mathrm{bc}$ & 1.187 & $0.385 \mathrm{c}$ & $0.403 \mathrm{~cd}$ & $7.13 b c$ & 7.42 & $2.41 \mathrm{c}$ & $2.52 \mathrm{~cd}$ \\
\hline & 40 & $1.182 \mathrm{abc}$ & 1.213 & $0.421 \mathrm{abc}$ & $0.443 a-d$ & 7.39abc & 7.58 & $2.63 \mathrm{abc}$ & $2.77 a-d$ \\
\hline & 80 & $1.207 \mathrm{abc}$ & 1.241 & $0.439 \mathrm{abc}$ & $0.456 \mathrm{a}-\mathrm{d}$ & $7.54 \mathrm{abc}$ & 7.76 & $2.75 \mathrm{abc}$ & $2.85 a-d$ \\
\hline & 120 & $1.237 \mathrm{abc}$ & 1.266 & $0.458 \mathrm{abc}$ & $0.486 a-d$ & $7.73 \mathrm{abc}$ & 7.91 & $2.87 \mathrm{abc}$ & $3.03 \mathrm{a}-\mathrm{d}$ \\
\hline \multirow[t]{4}{*}{ Phaseolus mungo } & 0 & $1.167 \mathrm{abc}$ & 1.203 & $0.412 \mathrm{abc}$ & $0.421 \mathrm{a}-\mathrm{d}$ & 7.29abc & 7.52 & $2.58 \mathrm{abc}$ & $2.63 a-d$ \\
\hline & 40 & $1.203 \mathrm{abc}$ & 1.225 & $0.422 \mathrm{abc}$ & $0.425 \mathrm{a}-\mathrm{d}$ & $7.52 \mathrm{abc}$ & 7.66 & $2.64 \mathrm{abc}$ & $2.66 \mathrm{a}-\mathrm{d}$ \\
\hline & 80 & $1.223 \mathrm{abc}$ & 1.238 & $0.451 \mathrm{abc}$ & $0.458 \mathrm{a}-\mathrm{d}$ & 7.64abc & 7.74 & $2.82 \mathrm{abc}$ & $2.86 \mathrm{a}-\mathrm{d}$ \\
\hline & 120 & $1.243 \mathrm{abc}$ & 1.269 & $0.470 \mathrm{abc}$ & $0.488 \mathrm{a}-\mathrm{d}$ & 7.77abc & 7.93 & $2.94 \mathrm{abc}$ & $3.05 \mathrm{a}-\mathrm{d}$ \\
\hline \multirow[t]{4}{*}{ Seasonal fallow } & 0 & $1.127 \mathrm{c}$ & 1.152 & $0.382 \mathrm{c}$ & $0.389 \mathrm{~d}$ & $7.04 \mathrm{c}$ & 7.20 & $2.39 \mathrm{c}$ & $2.43 \mathrm{~d}$ \\
\hline & 40 & $1.155 \mathrm{abc}$ & 1.186 & $0.397 \mathrm{bc}$ & $0.409 \mathrm{bcd}$ & $7.22 \mathrm{abc}$ & 7.41 & $2.48 \mathrm{bc}$ & $2.56 \mathrm{bcd}$ \\
\hline & 80 & $1.185 \mathrm{abc}$ & 1.209 & $0.426 \mathrm{abc}$ & $0.432 \mathrm{a}-\mathrm{d}$ & $7.41 \mathrm{abc}$ & 7.56 & $2.66 \mathrm{abc}$ & $2.70 a-d$ \\
\hline & 120 & $1.225 \mathrm{abc}$ & 1.23 & $0.444 \mathrm{abc}$ & $0.441 \mathrm{a}-\mathrm{d}$ & 7.66abc & 7.69 & $2.78 \mathrm{abc}$ & $2.76 a-d$ \\
\hline Level of significance & & 0.05 & NS & 0.01 & 0.05 & 0.05 & NS & 0.01 & 0.05 \\
\hline $\mathrm{CV}(\%)$ & & 6.16 & 7.84 & 10.23 & 12.8 & 6.15 & 7.84 & 10.19 & 12.8 \\
\hline
\end{tabular}


Md Safiqul Islam et al. / OnLine Journal of Biological Sciences 2015, 15 (4): 207.216 DOI: 10.3844/ojbsci.2015.207.216

Table 5. Influence of green manuring crops and nitrogen levels on N uptake by grain and straw of T.aman rice (cv. BRRI dhan 39)

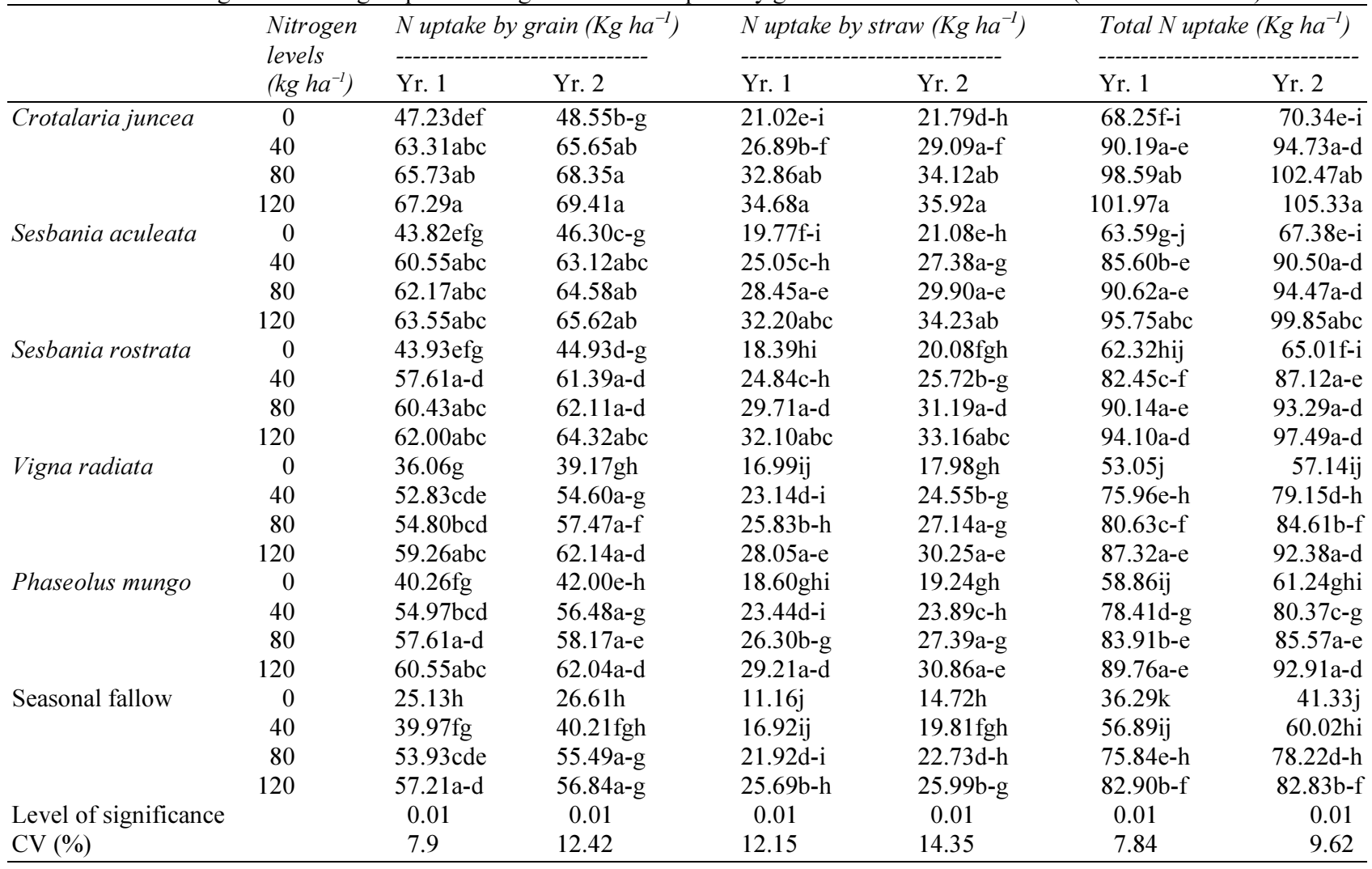

Table 6. Changes in post-harvest soil nutrient status as affected by green manures and nitrogen levels

\begin{tabular}{|c|c|c|c|c|c|c|c|c|c|c|c|c|c|c|c|}
\hline & \multirow{2}{*}{$\begin{array}{l}\text { Nitrogen } \\
\text { levels } \\
\mathrm{kg} \mathrm{ha}^{-1} \text { ) }\end{array}$} & \multicolumn{2}{|l|}{$\mathrm{pH}$} & \multicolumn{2}{|c|}{ Organic matter $(\%)$} & \multicolumn{2}{|c|}{ Total N (\%) } & \multicolumn{2}{|c|}{$\begin{array}{l}\text { Available P } \\
\left(\mathrm{mg} \mathrm{Kg}^{-1}\right)\end{array}$} & \multicolumn{2}{|c|}{ 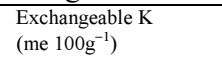 } & \multicolumn{2}{|c|}{$\begin{array}{l}\text { Available S } \\
\left(\mathrm{mg} \mathrm{Kg}^{-1}\right)\end{array}$} & \multicolumn{2}{|c|}{$\begin{array}{l}\text { Available } \mathrm{Zn} \\
\left(\mathrm{mg} \mathrm{Kg}^{-1}\right)\end{array}$} \\
\hline & & Yr. 1 & Yr. 2 & Yr. 1 & Yr. 2 & Yr. 1 & Yr. 2 & Yr. 1 & Yr. 2 & Yr. 1 & Yr. 2 & Yr. 1 & Yr. 2 & Yr. 1 & Yr. 2 \\
\hline \multirow[t]{4}{*}{ Crotalaria juncea } & 0 & 8.01 & 8.23 & 1.176 & $1.21 \mathrm{a}$ & $0.067 \mathrm{a}-\mathrm{d}$ & $0.071 \mathrm{a}-\mathrm{e}$ & 9.83 & 10.48 & $0.325 \mathrm{ab}$ & $0.290 \mathrm{~b}-\mathrm{f}$ & 22.39 & 22.55 & 0.513 & 0.558 \\
\hline & 40 & 8.01 & 8.26 & 1.152 & $1.17 \mathrm{abc}$ & $0.071 \mathrm{ab}$ & $0.075 \mathrm{a}-\mathrm{d}$ & 9.87 & 10.69 & $0.307 a b c$ & $0.392 \mathrm{a}$ & 22.57 & 22.89 & 0.492 & 0.537 \\
\hline & 80 & 8.02 & 8.29 & 1.160 & $1.15 \mathrm{abc}$ & $0.072 \mathrm{a}$ & $0.078 \mathrm{ab}$ & 10.16 & 10.58 & $0.325 \mathrm{ab}$ & $0.392 \mathrm{a}$ & 22.70 & 22.70 & 0.524 & 0.563 \\
\hline & 120 & 8.06 & 8.29 & 1.128 & $1.17 \mathrm{abc}$ & $0.074 \mathrm{a}$ & $0.080 \mathrm{a}$ & 10.03 & 10.78 & $0.343 \mathrm{a}$ & $0.358 \mathrm{ab}$ & 22.87 & 23.01 & 0.529 & 0.574 \\
\hline \multirow[t]{4}{*}{ Sesbania aculeata } & 0 & 8.02 & 8.25 & 1.160 & $1.18 \mathrm{ab}$ & $0.066 \mathrm{a}-\mathrm{d}$ & $0.069 \mathrm{a}-\mathrm{f}$ & 9.80 & 10.33 & $0.253 \mathrm{a}-\mathrm{d}$ & $0.256 \mathrm{c}-\mathrm{g}$ & 22.26 & 22.50 & 0.471 & 0.511 \\
\hline & 40 & 8.02 & 8.29 & 1.128 & $1.14 a b c$ & $0.069 \mathrm{a}-\mathrm{d}$ & $0.073 \mathrm{a}-\mathrm{d}$ & 9.69 & 10.45 & $0.289 \mathrm{a}-\mathrm{d}$ & $0.307 \mathrm{a}-\mathrm{e}$ & 22.55 & 22.32 & 0.492 & 0.532 \\
\hline & 80 & 8.10 & 8.32 & 1.120 & $1.16 \mathrm{abc}$ & $0.068 \mathrm{a}-\mathrm{d}$ & $0.075 \mathrm{a}-\mathrm{d}$ & 9.95 & 10.73 & $0.307 \mathrm{abc}$ & $0.341 \mathrm{abc}$ & 22.45 & 22.67 & 0.519 & 0.548 \\
\hline & 120 & 8.05 & 8.30 & 1.104 & $1.13 \mathrm{abc}$ & $0.070 \mathrm{abc}$ & $0.076 \mathrm{abc}$ & 10.07 & 10.60 & $0.307 \mathrm{abc}$ & $0.324 a-d$ & 22.79 & 22.87 & 0.508 & 0.537 \\
\hline \multirow[t]{4}{*}{ Sesbania rostrata } & 0 & 7.98 & 8.28 & 1.136 & $1.17 \mathrm{abc}$ & $0.065 \mathrm{a}-\mathrm{d}$ & $0.066 \mathrm{~b}-\mathrm{g}$ & 9.60 & 10.41 & $0.235 \mathrm{a}-\mathrm{d}$ & $0.238 \mathrm{~d}-\mathrm{g}$ & 22.15 & 22.12 & 0.460 & 0.495 \\
\hline & 40 & 8.05 & 8.25 & 1.104 & $1.15 \mathrm{abc}$ & $0.066 \mathrm{a}-\mathrm{d}$ & $0.073 \mathrm{a}-\mathrm{d}$ & 9.83 & 10.24 & $0.271 \mathrm{a}-\mathrm{d}$ & $0.290 \mathrm{~b}-\mathrm{f}$ & 21.98 & 22.30 & 0.476 & 0.526 \\
\hline & 80 & 8.01 & 8.28 & 1.112 & $1.14 a b c$ & $0.067 \mathrm{a}-\mathrm{d}$ & $0.071 \mathrm{a}-\mathrm{e}$ & 9.73 & 10.51 & $0.289 \mathrm{a}-\mathrm{d}$ & $0.307 a-e$ & 22.30 & 22.62 & 0.492 & 0.516 \\
\hline & 120 & 8.07 & 8.30 & 1.096 & $1.12 \mathrm{abc}$ & $0.067 \mathrm{a}-\mathrm{d}$ & $0.074 a-d$ & 9.98 & 10.65 & $0.289 \mathrm{a}-\mathrm{d}$ & $0.324 a-d$ & 22.45 & 22.47 & 0.497 & 0.532 \\
\hline \multirow[t]{4}{*}{ Vigna radiata } & 0 & 8.06 & 8.30 & 1.088 & $1.11 \mathrm{abc}$ & $0.054 \mathrm{de}$ & $0.059 \mathrm{efg}$ & 9.46 & 10.12 & $0.217 \mathrm{bcd}$ & $0.213 \mathrm{fg}$ & 21.83 & 21.96 & 0.436 & 0.468 \\
\hline & 40 & 8.06 & 8.35 & 1.084 & $1.09 \mathrm{abc}$ & $0.060 \mathrm{a}-\mathrm{e}$ & $0.061 \mathrm{~d}-\mathrm{g}$ & 9.57 & 10.13 & $0.226 a-d$ & 0.221 efg & 21.81 & 21.99 & 0.436 & 0.495 \\
\hline & 80 & 8.13 & 8.35 & 1.068 & $1.07 \mathrm{abc}$ & 0.059 a-e & $0.062 \mathrm{c}-\mathrm{g}$ & 9.82 & 10.32 & $0.217 \mathrm{bcd}$ & $0.230 \mathrm{efg}$ & 22.07 & 22.26 & 0.458 & 0.492 \\
\hline & 120 & 8.14 & 8.37 & 1.064 & $1.07 \mathrm{abc}$ & $0.060 \mathrm{a}-\mathrm{e}$ & $0.064 \mathrm{c}-\mathrm{g}$ & 9.69 & 10.45 & $0.244 a-d$ & $0.255 \mathrm{c}-\mathrm{g}$ & 22.09 & 22.27 & 0.466 & 0.502 \\
\hline \multirow[t]{4}{*}{ Phaseolus mungo } & 0 & 8.00 & 8.28 & 1.120 & $1.14 \mathrm{abc}$ & 0.059 a-e & $0.064 \mathrm{c}-\mathrm{g}$ & 9.53 & 10.22 & $0.235 \mathrm{a}-\mathrm{d}$ & 0.221 efg & 21.76 & 21.92 & 0.466 & 0.490 \\
\hline & 40 & 8.05 & 8.32 & 1.096 & $1.13 \mathrm{abc}$ & $0.064 \mathrm{a}-\mathrm{d}$ & $0.068 \mathrm{a}-\mathrm{g}$ & 9.55 & 10.10 & $0.271 \mathrm{a}-\mathrm{d}$ & $0.256 \mathrm{c}-\mathrm{g}$ & 22.02 & 22.20 & 0.450 & 0.516 \\
\hline & 80 & 8.09 & 8.33 & 1.080 & $1.10 \mathrm{abc}$ & 0.062 a-e & $0.067 \mathrm{a}-\mathrm{g}$ & 9.64 & 10.34 & $0.235 \mathrm{a}-\mathrm{d}$ & $0.256 \mathrm{c}-\mathrm{g}$ & 22.18 & 22.10 & 0.476 & 0.516 \\
\hline & 120 & 8.12 & 8.34 & 1.088 & $1.11 \mathrm{abc}$ & $0.064 \mathrm{a}-\mathrm{d}$ & $0.069 \mathrm{a}-\mathrm{f}$ & 9.83 & 10.46 & $0.271 \mathrm{a}-\mathrm{d}$ & $0.290 \mathrm{~b}-\mathrm{f}$ & 21.91 & 22.32 & 0.487 & 0.521 \\
\hline \multirow[t]{4}{*}{ Seasonal fallow } & 0 & 8.11 & 8.32 & 1.056 & $1.07 \mathrm{abc}$ & $0.049 \mathrm{e}$ & $0.055 \mathrm{~g}$ & 9.45 & 9.85 & $0.199 \mathrm{~cd}$ & $0.204 \mathrm{fg}$ & 21.52 & 21.83 & 0.407 & 0.447 \\
\hline & 40 & 8.07 & 8.38 & 1.072 & $1.06 \mathrm{bc}$ & $0.055 \mathrm{cde}$ & $0.056 \mathrm{fg}$ & 9.28 & 10.03 & $0.181 \mathrm{~d}$ & $0.187 \mathrm{~g}$ & 21.64 & 21.71 & 0.423 & 0.474 \\
\hline & 80 & 8.17 & 8.37 & 1.056 & $1.04 \mathrm{c}$ & $0.055 \mathrm{cde}$ & $0.058 \mathrm{efg}$ & 9.56 & 10.15 & $0.199 \mathrm{~cd}$ & $0.204 \mathrm{fg}$ & 21.84 & 21.92 & 0.439 & 0.469 \\
\hline & 120 & 8.15 & 8.41 & 1.039 & $1.03 \mathrm{c}$ & $0.056 \mathrm{~b}-\mathrm{e}$ & $0.059 \mathrm{efg}$ & 9.68 & 10.27 & $0.217 \mathrm{bcd}$ & 0.221 efg & 21.73 & 21.36 & 0.444 & 0.484 \\
\hline Level of significance & & NS & NS & NS & 0.05 & 0.05 & 0.01 & NS & NS & 0.01 & 0.01 & NS & NS & NS & NS \\
\hline CV $(\%)$ & & 5.93 & 5.2 & 7.08 & 6.01 & 11.95 & 9.27 & 12.36 & 11.59 & 14.4 & 10.81 & 12.91 & 12.57 & 13.97 & 10.89 \\
\hline
\end{tabular}

\section{Discussion}

We have evaluated the effect of both green manure (GM) and $\mathrm{N}$ application on T.aman rice in terms of crop growth, yield, and yield-contributing characters. The GM crops in situ were incorporated into the soil during land preparation a few days before transplantation of rice seedlings. Nitrogen at the rate of 40,80 , and $120 \mathrm{~kg} \mathrm{~N} \mathrm{ha}^{-1}$ from urea was applied in three equal installments at basal, tillering, and panicle initiation stages. There were also fallow plots where neither GM nor $\mathrm{N}$ was added.

In general, RLGR decreased with the advancement of plant age in both years. The negative RLGR values 
observed at the later stages were due to variation in treatment and age of the plant in both years. This might be due to more uptake of $\mathrm{N}$ at the early growth stages, resulting in more leaf area coverage and thereby producing more leaf dry weight, but abscission of mature leaves at the later stages of plant growth reduced leaf area. These results are similar to the findings of Alam and Haider (2006) in barley and of Rahman (2004) in wheat. More or less higher SLA values were found in the seasonal fallow treatment at most of the growth stages, and lower SLA values were found where GM crops were incorporated to the soil. SLA started with a maximum value of 30 DAT and declined with the progress of the growth period towards maturity. The decline of SLA with increasing plant age was noticed by Sarker and Paul (1998) in wheat. In both years, LWR increased for a very short time (30 DAT) and thereafter gradually declined with plant age. The decrease of LWR was caused by increased total dry matter (TDM) and decreased LAI at the later stages. Mollah (2007) studied LWR in wheat and reported that the sharp decrease in LWR at the later stages might be due to the sharp increase of TDM. Green leaf area is the source of food production in green plants. Leaf area index (LAI) increased steadily as the crop growth period advanced and reached its highest peak at 60 DAT and then started falling until the final harvest. Increases in LAI because of using GM might be due to the additional supply of $\mathrm{N}$ in the soil from the green manuring. Similar results were also reported by Hossain et al. (1995). The probable reason for the LAI increase in the nitrogen-treated plants is greater expansion of the leaf blades. The decrease in LAI at the later stages was possibly due to the senescence and abscission of the older leaves. These observations are in agreement with the findings of Hossain et al. (1995) and Pramanik (2006) in rice. CGR is regarded as the most meaningful growth function, since it represents the net results of photosynthesis, respiration, and canopy area interaction. CGR increased progressively with the age of the plants up to 45-60 DAT and thereafter decreased slowly. The decreasing trend might be due to leaf abscission at the later growth stages. This result agrees with the observations of Alam and Haider (2006) on barley. NAR decreased progressively with the age of the plants up to 75-90 DAT, perhaps due to mutual leaf shading from the increase in the number of older leaves that had lost photosynthetic ability, and thereafter it showed an increasing trend up to 90-105 DAT. Pramanik (2006) reported similar results in rice. NAR calculated from the quadratic fitted values showed that it decreased slowly with fluctuations in most cases, and thereafter declined sharply at the later stages of growth, in many cases reaching negative values.

The number of effective tillers hill ${ }^{-1}$ was significantly affected by the combination of green manure and nitrogen addition throughout the growth period of the crop. Nitrogen encouraged tiller production, and so the number of effective tillers plant ${ }^{-1}$ increased with the increase in Nfertilization. These observations are in agreement with the findings of Saha et al. (2007) in rice. Different green manure crops and nitrogen application had significant effects on the grains panicle ${ }^{-1}$ of $\mathrm{T}$. amanrice in each year of experimentation. Grains panicle $^{-1}$ of $\mathrm{T}$. aman in different green manuring plots was significantly higher than in the seasonal fallow and the plots with no nitrogen application. The increase in grains panicle ${ }^{-1}$ under GM might be due to the additional supply of $\mathrm{N}$ in the soil through green manuring. Pramanik (2006) found higher numbers of grains panicle ${ }^{-1}$ when $S$. rostrata was incorporated in the soil. These results are also in agreement with the findings of some other authors (Hossain et al., 1995; Bhander et al., 1998). Grain yield of T.aman rice is a complex variable depending upon a large number of environmental, morphological, and physiological characters. Grain yields also depend upon other yield components. In the present investigation, grain yield of T.aman rice was significantly influenced by the incorporation of green manure crops and nitrogen. The nutrients contributed through green manuring and nitrogen addition might be the reason for the increase in grain yield. These results are in agreement with the findings of Saha et al. (2007) and Islam et al. (2014). The combination of green manure and nitrogen had a significant effect on straw yield in both years. An increasing trend of straw yield was observed with higher levels of $\mathrm{N}$ in combination with green manures in both years. Saha et al. (2007) reported similar results in rice. The combination of green manure and $\mathrm{N}$ addition did not have a significant effect on harvest index in either year. A similar result was obtained by Pramanik (2006).

The maximum nitrogen and protein content in grain was produced when green manure crops were incorporated, and that amount was higher than during seasonal fallow and with higher doses of $\mathrm{N}$; the minimum nitrogen/protein result was observed when the crop was not fertilized at all. This result corroborates the findings of Salam (2005) in rice, Islam (2001) in wheat, and Hossain (2007) in mungbean. Significantly, an increase in nitrogen and protein content in straw was observed with higher levels of nitrogen in all the treatment combinations in both years, irrespective of green manuring. Pramanik (2006) noticed similar findings in rice. Significantly higher nitrogen uptake by grain and straw was found with the incorporation of all the GM crops compared to the seasonal fallow. Nitrogen contributed through green manuring might be the reason for increased nitrogen uptake by grain and straw. This finding is similar to that of Singh et al. (1996) in rice.

The increase in $\mathrm{N}$ content was directly related to biological $\mathrm{N}_{2}$ fixation and organic matter addition to the soil. This result is in agreement with the findings of Nur-E-Elahi (1991). With a few exceptions, a linearly 
increasing trend of soil $\mathrm{P}$ was observed with higher levels of nitrogen in all the treatment combinations, irrespective of the addition of GM crops. The slight variation observed between the treatments might be due to variation in the nitrogen levels. A similar result was obtained by Panda et al. (1994). Except in some cases, the combination of GM and nitrogen addition increased significantly the availability of $\mathrm{K}$ in the soil. This result is in agreement with the findings of Bellakki and Badanur (1997).

\section{Conclusion}

Crotalaria juncea, Sesbania aculeata and Sesbania rostrata were found to be effective green manure crops in terms of biomass, nodulation, and nitrogen contribution, and especially in the positive effect they exerted on the immediate and next crops in our ricewheat system. These plants, when incorporated into soil, along with urea application at $40 \mathrm{~kg} \mathrm{~N} \mathrm{ha}^{-1}$, produced higher and more satisfactory yields of T.aman rice than were obtained from the application of urea alone at $80 \mathrm{~kg} \mathrm{~N}$ $\mathrm{ha}^{-1}$. The GM addition also had a considerable positive effect, although not significant, on the following crop (wheat). The GM-amended soil also yielded higher protein content (\%) in rice grain. As observed from soil analysis (after one crop cycle), both organic matter and $\mathrm{N}$ content significantly increased in the GM-treated plots. Hence, for improvement and sustainability of soil fertility, and for crop productivity, an integrated management approach that combines the use of fertilizers and manures is essential.

\section{Acknowledgement}

The authors thanks to the Institute of Biological Science, University of Rajshahi for their technical assistance during the study period.

\section{Funding Information}

The authors gratefully acknowledge BARI authority for providing scholarship and research facilities.

\section{Author's Contributions}

Md. Shafiqul Islam: Performed the experiments and analyzed the data.

Nishit Kumar Paul: Designed the experiments and analyzed the data.

Md. Robiul Alam: Performed the experiments and analyzed the data.

Md. Romij Uddin: Wrote the manuscript and analyzed the data.

Uttam Kumer Sarker: Wrote the manuscript and analyzed the data.

Md. Ariful Islam: Performed the experiments and analyzed the data.

Sung Un Park: Designed the experiments and analyzed the data.

\section{Ethics}

This manuscript has not been published or presented elsewhere in part or in entirely, and is not under consideration by another journal. All the authors have approved the manuscript and agree with submission to your esteemed journal. There are no conflicts of interest to declare.

\section{References}

Aktar, M.S., M.K. Hossain, R.C. Adhekery and M.K. Chowdhury, 1993. Integrated management of Sesbania rostrata and urea nitrogen in rice under a rice-rice cropping system. Ann. Bangladesh Agric., 3: 109-111.

Alam, M.Z. and S.A. Haider, 2006. Growth attributes of barley (Hordeum vulgare L.) Cultivars in relation to different doses of nitrogen fertilizer. J. Life Earth Sci., 1: 77-82.

BARC, 2012. Fertilizer recommendation GUIDE-2012 soils pub. Bangladesh Agricultural Research Council.

Bellakki, M.A. and V.P. Badanur, 1997. Long-term effect of integrated nutrient management on properties of vertisol under dryland agriculture. J. Indian Soc. Soil Sci., 45: 438-442.

Bhander, P.K., M.S.U. Bhuiyan and M.A. Islam, 1998. Effect of Sesbania rostrata biomass and nitrogen fertilizer on the yield attributes of T.aman rice. Progress. Agri., 9: 89-93.

Hossain, M.A., A.B.M. Salauddin, S.K. Roy, S. Nasreen and M.A. Ali, 1995. Effect of green manuring on the growth and yield to transplant aman rice. Bangladesh J. Agril. Sci., 22: 21-29.

Hossain, M.I., 2007. Studies on productivity, soil fertility and N-use efficiency in ric-wheatmungbean systems using tillage options, straw management and nitrogen levels. Ph.D. Thesis, Dept. Agron. and Agril. Ext., Rajshahi University. Rajshahi. pp: 75-222.

Hunt, R., 1978. Plant Growth Analysis. Studies in Biology, No. 96. Edward Arnold (Publishes) Limited, 41. Bedford Square, London.

IARI, 1995. Investigation on sustainability of rice-wheat cropping system. In: NARP report on basic research on rice-wheat cropping system. Indian Agricultural Research Institute.

IRRI, 1988. The role of green manure in rice farming systems. International Rice Research Institute.

Islam, M.R., M.B., Hossain, A.B., Siddique, M.T., Rahman and M. Malika, 2014. Contribution of green manure incorporation in combination with nitrogen fertilizer in rice production. J. SAARC Agri., 12: 134-142.

Islam, M.S., 2001. Effect of irrigation and nitrogen on the growth, yield and quality of wheat. MSc Thesis, Bangladesh Agricultural University, Mymensingsh. 
Mollah, M.S.I., 2007. Studies on the physiological attributes and their relationship to yield of barley (Hordeum vulgare L.) under different soil moisture regimes and N P K fertilizers. PhD Thesis, Rajshahi University, Rajshahi.

Nur-E-Elahi, M., 1991. Intercropping Forage-Green Manure with a Grain Legume in the Pre-Rice DryWet Period for Food, Feed and Organic Nitrogen Across Irrigated and Rainfed Rice Ecosystems. 1st Edn., Cornell University, pp: 912.

Panda, M.M., A.R. Mosier, S.K. Mohanty, S.P. Chakravorti and A.B. Chalam et al., 1994. Nitrogen utilization by lowland rice as affected by fertilization with urea and green manure. Fert. Res., 40: 215-223. DOI: $10.1007 / \mathrm{BF} 00750468$

Pradhan, S.B., 1992. Status of fertilizer use in developing countries of asia and the pacific region. Proc. Reg. Fadinap Seminar. Chang Mai. Thailand.

Pramanik, M.Y.A., 2006. Effect of green manuring on transplant aman rice and its residual effect on subsequent boro rice. Ph.D. Thesis. Department of Agronomy, Bangladesh Agricultural University, Mymensingsh.

Radford, P.J., 1967. Growth analysis formulae-their use and abuse. Crop Sci., 7: 171-175. DOI:10.2135/cropsci1967.0011183X000700030001x

Rahman, M.M., 2004. Effect of irrigation on nitrogen uptake, yield and attributes of wheat varieties. Ph D. Thesis, Crop Physiology Laboratory, Department of Botany, Rajshahi University.
Rijpma, J. and M. Jahiruddin, 2004. National Strategy and plan for use of soil nutrient balance in Bangladesh: A consultancy report. SFFP, Khamarbari, Dhaka.

Saha, P.K., M. Ishaque, M.A. Saleque, M.A.M. Miah and G.M. Panaullah et al., 2007. Long-term integrated nutrient management for rice-based cropping pattern: Effect on growth, yield, nutrient uptake, nutrient balance sheet and soil fertility. Communications Soil Sci. Plant Anal., 38: 579-610. DOI: 10.1080/00103620701215718

Salam, M.A., 2005. Nutrient management for wheatfallow- t.aman rice cropping pattern in high barind track. M.S. Thesis. Dept. Soil Sci., Bangladesh Agricultural University, Mymensingsh, pp: 37-76.

Sarker, A.M. and N.K. Paul, 1998. Studies on the growth attributes of wheat under irrigated and rainfed conditions. Bangladesh J. Bot, 27: 119-126.

Singh, Y., B. Singh, O.P. Meelu and M.S. Maskina, 1990. Nitrogen equivalence of green manure for wetland rice on course textural soils. Int. Rice Res. Notes, 15: 25-25.

Singh, G., R. Singh and P. Kumar, 1996. Response of wheat (Triticum aestivum) to nitrogen, phosphorus and potassium fertilizer. Indian J. Agron, 41: 157-157.

Tiwari, K.N., A.N. Pathak and H. Ram, 1980. Green manuring in combination with fertilizer nitrogen in rice under double cropping system in alluvial soils. J. Indian Soc. Soil Sci., 28: 162-169. 